\title{
Preneoplastic Lesions Fimbria Early Diagnosis Markers Underlying Timeline Mechanisms at The Origin of Ovarian Cancer in BRAC1/2 Patients: Case Reports Based on Proteogenomic Study
}

\author{
Maxence Wisztorski \\ Université de Lille \\ Philippe Saudemont \\ Université de lille
}

\section{Soulaimane Aboulouard}

Université de Lille

\section{Tristan Cardon \\ Université de lille}

Fabrice Narducci

CLCC Oscar Lambret: Centre Oscar Lambret

\section{Yves-Marie Robin}

CLCC Centre Oscar Lambret: Centre Oscar Lambret

Anne-Sophie Lemaire

CLCC Centre Oscar Lambret: Centre Oscar Lambret

\section{Delphine Bertin}

CLCC Centre Oscar Lambret: Centre Oscar Lambret

\section{Firas Kobeissy}

American University of Beirut

\section{Eric Leblanc}

CLCC Centre Oscar Lambret: Centre Oscar Lambret

\section{Isabelle Fournier}

Université de Lille

Michel Salzet ( $\sim$ michel.salzet@univ-lille.fr)

Université de Lille: Universite de Lille https://orcid.org/0000-0003-4318-0817

\section{Research}

Keywords: Spatially resolved proteomic, Alternative proteins, Ghost proteome, Protein mutations, FFPE tissues, Fimbria, p53 signature, STIL, STIC, HGSC, ovarian cancer

Posted Date: January 25th, 2021

DOI: https://doi.org/10.21203/rs.3.rs-152452/v1 
License: (9) (1) This work is licensed under a Creative Commons Attribution 4.0 International License. Read Full License 


\section{Abstract}

Background : BRCA 1 or 2 mutations have been known to be drivers of high-grade serous ovarian carcinomas (HGSC). As no efficient screening is currently available, a bilateral prophylactic salpingo-oophorectomy is the recommended procedure to avoid this often lethal carcinomas. However, a proportion of BRCA mutation carriers refuse to undergo this procedure due to significant impacts on quality and quantity of life, especially if a hormone replacement therapy is contra-indicated. Such decision expose them to developing a pelvic serous carcinoma. A better undersanding of high-grade serous cancer pathogenesis, rather stemming from fallopian tubes than from ovary itself, makes interesting a two-step prophylactic strategy in which tubes are removed as soon as possible, followed by oophorectomies at the time of menopause. Thus women are protected against HGSC with no immediate need of an HRT. Following this idea and considering the concept of junctional "hot spots " (called mesothelo-müllerian junctions) for the development of HGSC, we suggested as first step to perform a bilateral prophylactic radical fimbriectomy rather than salpingectomy with a delayed oophorectomy (RF/DO). This operation was tested in a national phase 2 trial from 2011-2014 (NCT01608074). Radical Fimbriectomy (RF) consists of the surgical removal of both fallopian tubes along with a tiny part of the adherent ovary to suppress the tubal source of possible dysplastic cells from which can stem a high-grade invasive tumor, through stepwise cellular and genomic altereations ranging from p53 signatures to serous tubal intraepithelial carcoinoma (STIC), just before genuine invasive HGSC, through intermediate aspects (STIL) and (TILT).

Methods : In this context, we carried out an in-depth proteomics analysis of these "pre-invasive " epithelial lesions based on spatially resolved proteomic/ and bioinformatics systems biology platforms platform guided by immunohistochemistry (IHC) technique.

Results : Specific markers related to each preneoplastic lesions and in particular from normal to p53 signatures were evaluated. We identified EIF3B, MOB1B and Emilin2, CAVIN1 as bad prognosis markers of P53 signature whereas CAVIN2, SPTANi, FBLN5 as good prognosis markers. Among the novel markers identified, mutated proteins as well as hidden proteins translated from alternative ORF Proteome have been sequenced and characterized.

Conlusion : In summary, our results represent a novel pioneer approach to identify the unique under investigated markers that can characterize the molecular mechanism occurring in the preneoplastic lesion in fimbria at the origin of the HGSC.

\section{Background}

Ovarian carcinoma is rare (8th cause of feminine cancer), but from far one of the most lethal, with 295400 cases worldwide for 184800 deaths (GLOBOCAN, 2018). If the overall ovarian carcinoma affects 1/78 - 1/70 woman (1,2 $-1,4 \%)$ in the general population, the risk raises from $25 \%(11-27)$ to $45 \%(39-46)$ from $35-75$ years old, if this woman carries a BRCA 1 or 2 mutation respectively.

Whereas ovarian cancer $(\mathrm{OC})$ is ranked at the 8th position among cancers, it is by far the most lethal cancer; with an approximate of $30-40 \%$ survival rate in a period of five years. Amongst the different classes of varian cancers, high-grade serous carcinoma (HGSC) is the most represented (>75\%) having $15 \%$ of the patients with mutations in the BRCA1/2 gene (Braicu et al., 2011; Pal et al., 2005; Seidman et al., 2004). These patients exhibit a 20-40 percent increased risk of developing HGSC between 30 and 35 years old (versus $1.4 \%$ in the global population). 
During the last 10 years, special attention has been paid towards the pathological examination of operative specimens of risk-reducing salpingo-oophorectomy in women carrying a familial risk of OC. Most of the ovarian carcinomas related to BRCA 1 or 2 mutations are of invadsive high grade serous pathological subtype. They seem stem from the fallopian tube and particularly from its distal part called the fimbria..

Unfortunately as they are rapidly evolutive HGSC cannot be effectively screened. In addition if hormonal contraception seems efficient at preventing them, this treatment is sometimes not well tolerated or even contraindicated in the case of breast cancer history.

Risk-reducing surgery is therefore suggested to these high-risk ladies when child bearing project has benn completed. Bilateral risk-reducing salpingo-oophorectomy (RRSO) surgery is the gold standard prophylactic procedure worlwide.

Some BRCA-mutated women (or at-risk of ovarian cancer but without identified mutations) are reluctant to undergo this procedure considering the numerous adverse effects on the body and quality of life, especially when hormonal replacement is proposed. These woman are at higher rsk to develop serous pelvic carcinoma. The goal of the bilateral laparoscopic radical fimbriectomy is to suppress the tubal source of possible dysplastic cells from which high-grade tumor originates, while preserving a natural ovarian hormonal secretion. This procedure is offered only in case of rejection of BSO and it is aimed to salvage these high-risk women and keep them under adapted surveillance of their gonads. The reduction in benefits of castration over breast cancer risk, should be evaluated and assessed by the patinets. Complementary castration is recommended at 50 years old cohorts. This new prophylactic operation, based on ovarian carcinogenesis emergece, preserves femininity and possibly fertility as well. It seems an interesting option for selected women who are reluctant to undergo prophylactic BSO. Our work will evaluate Müllerian cells in the fimbria end of the fallopian tube to find a relationship between the pre-neoplastic lesions and (HGSC), a type II ovarian carcinoma. Based on Sectioning and Extensively Examining the Fimbriated End Protocol (SEE-FIM) (Mingels et al., 2014), a systematic serial examination of the fallopian tubes will be performed, coupled with IHC evaluation of the p53 and Ki-67protein expression. In this group of patients, an unusual rate of certain cancer or at least cellular abnormalities are observed in the fallopian tube epithelium (FTE), especially at its terminal end, the fimbria (Crum et al., 2007; Medeiros et al., 2006b). Three main lesions are defined according to the IHC pattern for the two markers and the architectural alterations of the cells. The p53 signature is identified by a small number of epithelial cells (10-20) with a proliferation activity like the adjacent normal epithelium, with a p53 staining pattern corresponding to a missense TP53 mutation. On the other hand, (serous tubal intraepithelial carcinoma (STIC) involves many cells with architectural and nuclear alterations, TP53 mutations and a high proliferative activity. While STILs (serous tubal intraepithelial lesions (STILS) are characterized by a lower level of abnormalities compared to the STIC along with a normal proliferative activity (Vang et al., 2012; Visvanathan et al., 2011a). accordingly, morphological changes concomitant with multi-step accumulation of molecular and genetic alterations on the pre-neoplastic lesions of FTE suggest that HGSCs derive their origin, not in the ovary, but from the fimbria part of the fallopian tube (Auersperg, 2013; Kobayashi et al., 2017; Roh et al., 2010). In fact PAX8, a Mullerian marker has been found to be expressed in most of the HGSC but not calretinin (mesothelial marker) (Bowtell, 2010; Folkins et al., 2009; Mingels et al., 2014). This view has not been universally accepted, primarily as it conflicts with traditional theories of the OC origins coupled with variation in the detection of tubal lesions in association with HGSC, which may, be due to differences in sampling or difficulties in diagnostic interpretation (Bachert et al., 2020; Singh et al., 2015). Recently, advanced studies have provided a stepwise progression of FTE to precursor lesions to carcinoma, with the aid of - p53 signature - STIL - STIC HGSC sequence's model (Kobayashi et al., 2017; Wu et al., 2019; Zeppernick et al., 2015). 
In this context, we performed a pan-proteomic study based on the state-of-the-art of the spatially resolved proteomics that have been previously applied on different kinds of cancers (Delcourt et al., 2017; Le Rhun et al., 2015; Wisztorski et al., 2016; Wisztorski et al., 2013; Wisztorski et al., 2017). This spatially resolved proteomic study is guided by IHC analysis performed on formalin-fixed paraffin-embedded (FFPE) tissue section of patients presented with p53 signature lesion, STIL, and STIC lesions compared to non-pathological fallopian tissues and HGSCs. Here, we investigate the different pre-neoplastic lesions, their proteome profile, their protein mutations landscape as well as assessing alternative ghost proteome translated from alternative ORF, and coupled with a bioinformatics sytems biology analysis to construct proteome molecular pathways indicative of the pathological underlying mechanisms (Cardon et al., 2020; Cardon et al., 2019; Delcourt et al., 2018; Vergara et al., 2020). By applying our approach, we identified the several early-stage markers such as EIF3B, MOB1B and Emilin2, CAVIN1 as bad prognosis markers and CAVIN2, SPTANi, FBLN5 as good prognosis markers. In addition, we have surveyed the molecular mechanisms occurring across pre-neoplastic lesions and confirm the benefit of the radical frimbioectomy in BRCA1/2 mutated patients .

\section{Experimental Procedures}

\section{Experimental Design and Statistical Rationale.}

Shotgun proteomics experiments were conducted on formalin-fixed paraffin-embedded (FFPE) tissue section of patients presented with p53 signature lesion, STIL lesions and STIC lesions which were compared to nonpathological fallopian tissues and HGSC samples; all experiments were conducted in biological triplicate. Statistical analysis: For the proteomics statistical analysis of extracted proteins or secreted media, only proteins presenting as significantly determined by the ANOVA test were used with an FDR of 5\%. Normalization was achieved using a Zscore with a matrix access by rows. Obtained data from Western blot were reported as mean \pm SEM. Mean values among different experimental groups were statistically compared by one-way ANOVA tests using Graph pad PRISM software or by student t-test.

\section{Reagents and Chemicals}

For the different experiments, we used high purity chemicals from various suppliers: HPLC grade methanol (MeOH), ethanol (EtOH), Chloroform ( $\mathrm{CHCl} 3)$, acetonitrile ( $\mathrm{ACN})$, water and trifluoroacetic acid (TFA) were obtained from Thermo Fisher Scientific (Courtabœuf, France). Trifluoracetic acid (TFA, 99\%), and ammonium bicarbonate (NH4HCO3) were purchased from Sigma-Aldrich (Saint-Quentin Fallavier, France). Xylene and formic acid (FA, $\geq$ 96\%) were purchased from Biosolve (Dieuze, France). Sequencing grade modified porcine trypsin was obtained from Promega (Charbonnieres, France).

\section{Surgery Procedure}

BRCA1/2 carriers or any women with a documented familial risk of breast/ovarian cancer were first counseled to perform a classical laparoscopic RRSO. If they denied, they were offered to enter the RF/DO trial. All specimens were submitted to the Sectioning and Extensively Examining-the FIMbriated end (SEE-FIM) pathological protocol. Pathological data along with all intra- and 30-day and beyond post-operative adverse events were prospectively recorded. Follow-up visit consisted of an annual clinical breast and gynecological examination, with tumor markers and hormonal status assessment. Primary endpoint was the rate of pelvic serous carcinoma. Secondary endpoints were procedure morbidity, rates of tubal abnormalities, breast cancer, secondary oophorectomy. An IRB Protocol NCT-01608074 was approved by the national and institutional review boards (IRB) and accepted by our local ethics 
committee. An interim analysis by a French DSMB was performed in January 2014. It was decided to complete the study until its end, without protocol modifications. This national phase 2 prospective study, started in 2012, ended in October 2014 after accrual completion. Eleven French centers actively participated in patients' recruitment.

\section{Case selection and sample processing}

Tissue samples were obtained from patients of the Centre Oscar Lambret (Lille, France. All experiments were approved by the local Ethics Committee (CPP Nord Ouest IV 12/10) in accordance with the French and European legislation. Prior to the experiments, patients signed an informed consent and authorization form describing the experimental protocol. No personal information was used in these experiments, and a random number was assigned to each sample. In our study, all samples were FFPE tissue from prophylactic adnexectomies of women with BRCA mutations. Our study was performed on patients presenting with pre-neoplastic lesions without any concomitant ovarian high-grade serous carcinoma. All samples were examined following SEE-FIM (Sectioning and Extensively Examining the FIMbria) protocol (Medeiros et al., 2006a). On these samples, tissues sections of $7 \mu \mathrm{m}$ thickness were cut using a microtome and were deposited on a glass slide, HPS (Haematoxylin Phloxine Saffron) and immunostaining against P53 and Ki-67 (Dako, Japan) were performed and examined by a pathologist to find preneoplastic lesions (p53/STIL/STIC/HGSC) (Visvanathan et al., 2011b). A cohort of eight patients was selected. Four patients presented p53 signature lesion, 2 patients with STIL lesions, 2 patients with STIC lesions, 1 patient with HGSC (see Table 1). The normal tissue was analyzed from the normal part (presenting no abnormalities at $\mathrm{IHC}$ ) of the tissue section from patients with p53 signature lesion. 
Table 1

description of clinical characteristics of patients with preneoplastic lesion in the fallopian tube fimbria.

\begin{tabular}{|c|c|c|c|c|c|c|c|c|}
\hline $\begin{array}{l}\text { Case } \\
\text { ID }\end{array}$ & $\begin{array}{l}\text { Lesions } \\
\text { sampled }\end{array}$ & $\begin{array}{l}\text { BRCA } \\
\text { status }\end{array}$ & $\begin{array}{l}\text { Age at } \\
\text { diagnosis }\end{array}$ & $\begin{array}{l}\text { Location } \\
\text { in } \\
\text { fimbria }\end{array}$ & $\begin{array}{l}\text { Ovary } \\
\text { lesion }\end{array}$ & $\begin{array}{l}\text { Other } \\
\text { lesions }\end{array}$ & $\begin{array}{l}\text { Personal } \\
\text { history of } \\
\text { breast cancer }\end{array}$ & $\begin{array}{l}\text { Other } \\
\text { remarks }\end{array}$ \\
\hline 1 & $\begin{array}{l}\text { Normal, } \\
\text { p53 } \\
\text { signature }\end{array}$ & BRCA1 & 40 & Right & No & $\begin{array}{l}\text { SCOUT in } \\
\text { tube }\end{array}$ & No & $\begin{array}{l}\text { Left } \\
\text { ovarian } \\
\text { theca cyst }\end{array}$ \\
\hline 2 & $\begin{array}{l}\text { Normal, } \\
\text { p53 } \\
\text { signature }\end{array}$ & BRCA1 & 39 & $\begin{array}{l}\text { Right } \\
\text { and left }\end{array}$ & No & No & Yes & \\
\hline 3 & STIL (x3) & unknown & 61 & left & No & No & No & $\begin{array}{l}\text { Uterus } \\
\text { Leiomyoma }\end{array}$ \\
\hline 4 & STIC (x3) & BRCA1 & 36 & $\begin{array}{l}\text { Right } \\
\text { and left }\end{array}$ & No & $\begin{array}{l}\text { Several } \\
\text { p53 } \\
\text { signature }\end{array}$ & No & $\begin{array}{l}\text { HGSC in } \\
\text { the left tube }\end{array}$ \\
\hline 5 & $\begin{array}{l}\text { Normal, } \\
\text { p53 } \\
\text { signature }\end{array}$ & $\begin{array}{l}\text { Family } \\
\text { history }\end{array}$ & 38 & Left & No & No & No & \\
\hline 6 & $\begin{array}{l}\text { STIL, } \\
\text { STIC }\end{array}$ & BRCA1 & 48 & Left & No & $\begin{array}{l}\text { Several } \\
\text { p53 } \\
\text { signature }\end{array}$ & Yes & \\
\hline 7 & $\begin{array}{l}\text { Normal, } \\
\text { p53 } \\
\text { signature }\end{array}$ & BRCA1 & 35 & Left & No & $\begin{array}{l}\text { Several } \\
\text { p53 } \\
\text { signature }\end{array}$ & yes & $\begin{array}{l}\text { HGSC in } \\
\text { the left } \\
\text { fimbria }\end{array}$ \\
\hline
\end{tabular}

\section{On-tissue spatially resolved proteomics}

A complete workflow for sample preparation is illustrated in Fig. 1A

\section{Sample preparation}

The same slides as the one used and annotated by the pathologist were unmounted; resin was removed by soaking them overnight in xylene and rinsing them with xylene and ethanol baths. The tissues were rehydrated using $5^{\prime}$ each successive bath of decreasing ethanol degree $\left(2 \times 95^{\circ}, 1 \times 30^{\circ}\right)$ and two baths of $10 \mathrm{mM} \mathrm{NH} 4 \mathrm{HCO} 3$ buffer. Then, an antigen retrieval step was performed to increase the trypsin access to biomolecules. For this, the slides were dipped in $90^{\circ} \mathrm{C} \mathrm{pH} 920 \mathrm{mM}$ Tris for 30 minutes, rinsed twice with $\mathrm{NH} 4 \mathrm{HCO} 3$, and dried under vacuum at room temperature.

In situtrypsin digestion

On localized pre-neoplastic lesions tryptic digestion was performed using a Chemical Inkjet Printer (CHIP-1000, Shimadzu, Kyoto, Japan). The trypsin solution (40 $\mathrm{g} / \mathrm{mL}, 50 \mathrm{mM} \mathrm{NH4HCO3} \mathrm{buffer)} \mathrm{was} \mathrm{deposited} \mathrm{on} \mathrm{a} \mathrm{region}$ defined to $1 \mathrm{~mm}^{2}$ during $2 \mathrm{~h}$. During this time, the trypsin was changed every half-hour. With $350 \mathrm{cycles}$ and $450 \mathrm{pL}$ per spot, a total of $6.3 \mu \mathrm{g}$ of trypsin was deposited. To stop digestion, $0.1 \%$ TFA was spotted during 25 cycles. 


\section{Liquid Extraction}

After microdigestion, the spot content was gathered by liquid microjunction using the TriVersa Nanomate (Advion Biosciences Inc., Ithaca, NY, USA) using Liquid Extraction and Surface Analysis (LESA) settings. With 3 different solvents mixture composed of $0.1 \%$ TFA, ACN/0.1\% TFA (8:2, v/v), and MeOH/0.1\% TFA (7:3, v/v). A complete LESA sequence run 2 cycles for each mixture composed of an aspiration $(2 \mu \mathrm{L})$, a mixing onto the tissue, and a dispensing into low-binding tubes. For each interesting spot, 2 sequences are pooled into the same vial.

\section{MS-based proteomic}

\section{NanoLC-ESI-MS}

After the liquid extraction, samples were freeze-dried in a SpeedVac concentrator (SPD131DPA, Thermo Scientific, Waltham, Massachusetts, USA), reconstituted with $10 \mu \mathrm{L} 0.1 \%$ TFA and subjected to solid-phase extraction to remove salts and concentrate the peptides. This was done using a C-18 Ziptip (Millipore, Saint-Quentin-en-Yvelines, France), eluted by an ACN/0.1\% TFA (8:2, v/v) and then the samples were dried for storage. Before analysis, samples were suspended in $20 \mu \mathrm{L} A C N / 0.1 \% \mathrm{FA}(2: 98, \mathrm{v} / \mathrm{v})$, deposited in vials, and 10 $\mathrm{LLs}$ were injected for analysis. The separation, prior to MS, used online reversed-phase chromatography realized with a Proxeon Easy-nLC-1000 system (Thermo Scientific) equipped with an Acclaim PepMap trap column (75 $\mu \mathrm{m} \mathrm{ID} \mathrm{x} 2 \mathrm{~cm}$, Thermo Scientific) and C18 packed tip Acclaim PepMap RSLC column (75 $\mu \mathrm{m}$ ID x $50 \mathrm{~cm}$, Thermo Scientific). Peptides were separated using an increasing amount of acetonitrile (5\%-40\% over 140 minutes) with a flow rate of $300 \mathrm{~nL} / \mathrm{min}$. The LC eluent was electrosprayed directly from the analytical column and a voltage of $2 \mathrm{kV}$ was applied via the liquid junction of the nanospray source.

The chromatography system was coupled to a Thermo Scientific Q-Exactive mass spectrometer. The mass spectrometer was programmed to acquire in a data-dependent mode for the 10 most intense peaks. The survey scans were acquired in the Orbitrap mass analyzer operated at 70,000 (FWHM) resolving power. A mass range of 200 to $2000 \mathrm{~m} / \mathrm{z}$ and a target of $3 \mathrm{E} 6$ ions were used for the survey scans. The MSMS analysis was performed using HCD with a normalized collision energy of $30 \mathrm{eV}$, a mass range between 200 to 2000, an AGC of 5e4 ions, a maximum injection time of $60 \mathrm{~ms}$ and a resolution set at 17,500 FWHM. The method was set to analyze the top 10 most intense ions from the survey scan and dynamic exclusion was enabled for $20 \mathrm{~s}$.

\section{Data Analyses}

MS/MS spectra were processed using MaxQuant (version 1.5.6.5, (Cox and Mann, 2008; Tyanova et al., 2015)). Peptides identification were obtained according to target decoy search against Homo sapiens Uniprot database (version of 2017_02, 20,172 sequences) and database containing 262 commonly detected contaminants. The human uniport database was used as the forward database and a reverse one for the decoy search was automatically generated in MaxQuant Mass tolerances were set to $10 \mathrm{ppm}$ and $20 \mathrm{ppm}$ respectively for parent and fragments measurement. Enzyme specificity was set to "trypsin" with a maximum of 2 missed cleavages allowed. Methionine oxidation and acetylation of protein N-terminal were set as variable modifications. FDR $<1 \%$ was set for peptides and proteins identification. Two peptides with one unique were necessary to assess protein identification. For label-free quantification, the MaxLFQ algorithm was used (Cox et al., 2014). The option "Match Between Runs" was enabled to maximize the number of quantification events across samples. This option allowed the quantification of high-resolution MS1 features not identified in every single measurement. Data generated by MaxQuant were analyzed using Perseus (version 1.6.2.3, (Tyanova and Cox, 2018). LFQ values were used and 
proteins were removed if found in the category only identified by site modifications, in the decoy reverse database, or identify in the contaminant database. We removed proteins that were not presented in at least three of four replicates. We took the average expression per group to perform a comparison and visualized using a Venn diagram. Individual LFQ values were used to perform a multi scatter plot and calculate a Pearson Correlation between samples. Missing values were imputed based on normal distribution (width $=0.3$, down-shift $=1.8$ ). Principal component analysis (PCA) was done to compare the protein content of each sample. An ANOVA Multisample test was performed and consolidated by a Permutation-based FDR (FDR $<0.05,250$ randomizations). A specific comparison between normal and p53 signature samples was performed using a student's T-test.

Proteins with significant differences were filtered out and values were z-scored. The samples were then clustered according to a Euclidean average as a distance measure for column and row clustering.

Up-regulated and down-regulated proteins in the different groups were used to perform an annotation analysis of gene ontology terms by using Funrich (v3.1.3) (Pathan et al., 2015). A hypergeometric test was performed against all annotated gene/protein list by comparing the multiple datasets. Enrichment for biological process, transcription factor and cellular component were present as a bar chart. PANTHER Classification System (v14.1) was also used. PANTHER Overrepresentation test (Released 20190701) was performed using each list of up-or down-regulated proteins as "analyzed list" and Homo sapiens as "reference list". Fisher's Exact test with false discovery rate correction was used.

Subnetwork Enrichment Analysis (SNEA) from Elsevier's PathwayStudio version 10.0 (Elsevier) (Bonnet et al., 2009; Yuryev et al., 2009) was used to extract statistically significant altered biological and functional pathways in the different clusters of proteins.

The immunohistochemical $(\mathrm{IHC})$ data of different proteins of interest were investigated from the Human Protein Atlas (HPA) database (http://www.proteinatlas.org, (Uhlen et al., 2017)). Evaluation of the prognostic effects of these proteins on the overall survival (OS) was also extracted from HPA. Comparisons are performed with the 20 genes of highest significance associated with unfavorable prognosis for ovarian cancer, cervix cancer, endometrial cancer, and breast cancer.

\section{Mutation identification:}

The MS data were also processed to search for potential mutated peptides using the XMAn database (Yang and Lazar, 2014). This database contains information on mutations observed in cancers and diseases. Proteome Discoverer 2.1 was used to query the data using MS Amanda as a search node against the XMAn database, the human Uniprot database, and a database containing potential contaminants. Peptides identified only in the XMAn database at a high level of confidence were selected, and the MSMS spectra were manually inspected to confirm the presence of the mutation.

\section{Alternative Proteins identification}

RAW data obtained by nanoLC-MS/MS analysis were analyzed with Proteome Discoverer V2.3 (Thermo Scientific) using the Label-Free Quantification node, the protein database is downloaded from Openprot

(https://openprot.org/)(Brunet et al., 2019). This database included RefProt, novel isoforms, and AltProts predicted from both Ensembl and RefSeq annotations (Ensembl: GRCh38.83, RefSeq: GRCh38p7) for a total of 658,263 entries. The following processing and consensus parameters are used with: Trypsin as an enzyme, 2 missed cleavages, methionine oxidation as variable modification, precursor mass tolerance: $10 \mathrm{ppm}$, and fragment mass 
tolerance: 0.1 Da. The validation was performed using Percolator with a protein strict FDR set to 0.001. A consensus workflow was then applied for the statistical arrangement, using the high confidence protein identification. Results are filtered to keep master protein and high confidence protein FDR. Then results are extract in table, in order to use the LFQ values in PERSEUS, where an ANOVA statistical test is performed and the results represented by a diagram, like heatmap has described above.

\section{Results}

\section{Clinical Cohorts}

120 radical frimbriectomy (RF) were performed (clinical trial, NCT01608074) by laparoscopy where no patient developed any pelvic HGSC.

\section{Spatially-resolved proteomic guided by p53 and Ki67 IHC}

From this cohort, as cases reports, 8 patients were selected to represent the different preneoplastic lesions (p53 signature, STIL and STIC lesions) for the experiments (see Table 1). We used immunostained tissue sections obtained from the SEE-FIM protocol (Fig. 1A). This protocol consisting of systematic examination of the totality of the fimbria is difficult to perform and will not allow observation of a lot of pre-cancerous lesions. Indeed, the number of samples is then critically limited allowing minimal slide examination. Moreover, the pre-cancerous lesions presented an extremely limited number of cells on the epithelial layer with no specific morphological features (Fig. 1B). The presence of p53 signature, STIL, and STIC lesions were; thus, confirmed- thanks to a double IHC against p53 and Ki-67 markers (Fig. 1B). In order to process these samples for spatially resolved proteomics, we developed a new protocol directly using the same IHC slide used by the pathologist which has served to the detection of the lesions. The cover slide and the mounting medium were removed by extensive bathing of xylene and ethanol. A visual inspection was carried out to check whether additional washing is required to remove all the mounting medium from the tissue section. One critical point was the antigen retrieval procedure that needed to be performed after tissue rehydration. Next, the region of interest corresponding to a lesion is covered with a droplet of trypsin solution. The resulting tryptic peptides are extracted from the tissue by a droplet-based liquid microextraction derived from the LESA method (Quanico et al., 2016; Quanico et al., 2013; Wisztorski et al., 2016; Wisztorski et al., 2013; Wisztorski et al., 2010; Wisztorski et al., 2017) and analyzed by MS-based proteomics. Considering all the pre-neoplastic lesions and non-pathological tissues, 10375 unique peptide sequences corresponding to 1617 distinct protein groups have been identified using our workflow. MaxLFQ algorithm was used to perform label-free quantification of proteins and resulted in a total of 1571 protein quantifications. After filtering one according to a minimum number of values (2/3 of valid values) in at least one group of the four defined groups, 1046 proteins were obtained. This result is like those obtained in our previous experiments on FFPE tissue. Furthermore, using immunostained tissues, after removing the coverslip glass and the mounting medium, did not appear to affect not affect the microproteomic analysis.

To confirm these findingss, we performed a correlation analysis based on all quantified proteins. Proteomes inside samples from the same group were similar (mean Pearson correlation 0.92) compared with inter-group variation. The most differences were observed between STIC and STIL lesions (mean of 0.84) (Fig. 2A). This is in accordance with what is generally observed considering the inter-patient variability, and the protocol we used did not appear to alter the proteomic content of the tissue sections. These results allowed us to perform a comparative analysis to obtain a specific proteomic signature of the different lesions. 


\section{Spatially resolved proteomic study of p53 signature, STIL, STIC, and normal Fimbria}

Comparing the content of each group by averaging the replicates and using a Venn diagram representation, 853 common proteins (81.5\% of overlap) were observed between normal tissues and pre-neoplastic lesions (Fig. 2B, Supp. Data 1). Few unique proteins in each group were obtained. One protein is found only in the p53 signature lesion and three exclusives to the STIC lesions. The protein RNA-binding protein 10 (RBM10), found in three of the four p53 signature lesions and not in the normal tissue or other lesions, is an RNA-binding protein located in the nucleus. Human Protein Atlas (HPA) data showed that the overexpression of this gene is favorable in terms of prognostic in the case of cervical cancer and endometrial cancer. Three proteins (Glucosamine ( $\mathrm{N}$-acetyl)-6sulfatase (GNS), Upstream binding transcription factor, RNA polymerase I (UBTF) and ATPase family, AAA domain containing $3(A T A D 3 A / B)$ ) are exclusives to STIC lesions. Survival data extracted from HPA showed that elevated expression of GNS and ATAD3A/B are not favorable for survival in liver cancer. GNS is also a poor prognosis for glioma and presents the same tendency in ovarian cancer. The expression of UBTF appears to have no impact on survival. The inclusion of HGSC data in the analysis (Fig. 2C, Supp data 2), revealed that UBTF is present in STIC lesion and in the HGSC. Fibrillarin (FBL) is found only in the pre-cancerous lesions and not in the normal tissue. This protein is also observed in HGSC (Fig. 2C, Supp. Data 2). Comparison between the data of the different lesions and the HPA list of the 20 genes of highest significance associated with unfavorable prognosis in ovarian cancer revealed only two proteins (TGFBI and RPL7A), 3 (SUMF2, TGFBI, FASN) with cervix cancer, 1 with endometrial cancer (ASS1) and 4 (PGK1, ATP5F1B, TAGLN2, RAB5C) with breast cancer.

To better understand the modulation registered across the different lesions, principal component analysis (PCA) and a hierarchical clustering analysis were performed to visualize the correlation between samples. PCA based on whole proteome levels allow visualization of the difference among each group. (Fig. 2D). Components 1 and 2 account for $42.5 \%$ of the total data variation. Samples from normal tissue and p53 signature are closed and could not be easily differentiated. Samples from STIL and STIC lesions differed from normal and p53 signature samples in component 2. STIL lesions were closer to normal/p53 lesions in component 1 than STIC. Adjunction of the HCSG data confirmed the separation of the nature of the proteome between p53 signature, STIL, STIC and HGSC (Fig. 2D). Hierarchical clustering showed a significant difference in expression between the 5 groups as shown in the heatmap (Fig. 2E). Two main branches separate HGSC from the other lesions. The second branch is subdivided again into two subbranches i.e., one separating the STIC to p53 signature/Normal/STIL (Fig. 2E). The second subbranches were then separated between STIL to p53 signature/Normal. The last group (p53/Normal) was difficult to be differentiated. Nevertheless, the difference in cell composition between the ovary and the fallopian tube did not allow a direct conclusion to be drawn on the relationship between HGSC and the pre-neoplastic lesions observed in the fallopian tube. However, we could observe that the proteomic content of the STIC lesions is closer to HGSC than to the other lesions and that each lesion seemed to present a specific proteomic signature.

\section{Proteomic analyses along the tumoral process}

For a deeper analysis of the pre-neoplastic lesions of the FTE, ANOVA testing was performed without the HGSC data. A total of 197 proteins showed a variation of expression on the four groups (Supp. Data 3). A hierarchical cluster based on the expression of these proteins resulted in three groups and two mains clusters (Fig. 3A, Supp.

Data 3). As observed previously in PCA, normal and p53 signature could not be resolved and formed one group. This group and another group composed of the STIL lesions samples clustered together. The proteomic signature of STIC differed from the two previous groups and samples from STIC lesions formed the second cluster 
suggesting that proteomics profiles differed a lot from the two other groups. The heatmap presents five main clusters of proteins. Proteins that were enriched in STIL and STIC regions were represented in cluster 1. Those that were more abundant in both normal tissue and p53 signature lesions were represented in cluster 2 , proteins presented in normal, p53 signature lesion and STIL but not in the STIC were represented in cluster 3. Proteins that were specifically overexpressed in the STIC were represented in cluster $\mathbf{4}$ and those specific to normal tissue, p53 signature lesion, and STIC were represented in cluster 5. Cluster 1 (STIL/STIC) was composed of only 5 proteins i.e., MARCKS-related protein (MARCKSL1), Myosin-10 (MYH10), Protein SET (SET), Double-stranded RNA-binding staufen homolog-1 (STAU1), and D-3-phosphoglycerate dehydrogenase (PHGDH). This SET of proteins participates in numerous cellular functions including DNA repair, transcription, cell survival and proliferation. This protein is also involved in many cancer processes such as metastasis, the development of therapeutic drug resistance and plays a key role in tumorigenesis (Bayarkhangai et al., 2018). MARCKSL1 is known to promote the progression of lung adenocarcinoma by regulating Epithelial-mesenchymal transition (EMT) (Liang et al., 2020). STAU1 has been previously identified in colorectal cancer (Zhu et al., 2005), whereas PHGDH protein has been identified in ovarian cancer (Francavilla et al., 2017; Zhang et al., 2018). In cluster 2 (normal-p53), 15 proteins showed an overexpression for normal and p53 signature lesion. Among these proteins, CD166 antigen (ALCAM/CD166) is shown to be a potential cancer stem cell marker (Shimamura et al., 2014). It has been found as a poor prognosis marker in pancreatic cancer (Kahlert et al., 2009) or gastric cancer (Levin et al., 2010). This protein is known to contribute to local invasion and tumor progression by acting on the detachment of tumor cells (Lunter et al., 2005; Ofori-Acquah and King, 2008). Moreover, TNP01, ACLY, NME1, FLOT1, KLC4 are proteins already know to be involved in epithelial ovarian cancer (Creekmore et al., 2011; Kalra and Bapat, 2013; Li et al., 2018). Gene Ontology (GO) analyses confirmed that these proteins are involved in cell proliferation, adhesion, and growth (Fig. 3Ba). Cluster $\mathbf{3}$ (normalp53/STIL) contains 80 proteins. Proteins involved in complement and coagulation cascades are present in this cluster (SERPIN A1, SERPIN C1, SERPIN G1, C4a, C9, Vitamin D-binding protein (GC), pro-thrombin (F2), Vitronectin (VTN), kininogen (KNG1)) and 10 members of the collagen family. The PGRMC1 (Membrane-associated progesterone receptor component 1) is known to be involved in ovarian cancer (Peluso, 2011) as well as PTGIS (Prostacyclin synthase) (Santin et al., 2004) and the Protein NDRG1 known to modulate genes involved in ovarian cancer metastasis (Zhao et al., 2011). GO analyses confirmed that proteins are involved in neoplasia/metastasis, angiogenesis, and apoptosis (Fig. 3Bb). Cluster 4 (STIC only) contained 36 proteins where proteins involved in cell migration can be identified such as CFL1 (Cofilin-1), PFN1 (profilin-1), PPL (periplakin), FLNB (filamin B), PDLM1(PDZ and LIM domain protein 1), IQGAP1 (Ras GTPase-activating-like protein), MYH14 (Myosin-14) and in metabolism (CS (Citrate synthase), DECR1 (2,4-dienoyl-CoA reductase), GSR (Glutathione reductase), LAP3 (Cytosol aminopeptidase), GLUD1 (Glutamate dehydrogenase 1). Proteins of this cluster were involved in cell differentiation and proliferation and still in neoplasia/metastasis (Fig. 3Bc). The last cluster (normal-p53/STIC) was composed of 61 proteins. Several proteins involved in exosomes have been identified (Annexins A1, A2, A4, A13, EZR (ezrin), HSPA8 (Heat shock 71kDa protein 1A)). other proteins are involved in stress (PRDX1 'Peroxiredoxin-1), HSP8, HSPA1 (Heat shock 70 kDa protein 1), HSPA2 (Heat shock 70 kDa protein 2), ST13 (Hsc70-interacting protein), DNAJB6 (DnaJ homolog subfamily B member 6), VCP (Transitional endoplasmic reticulum ATPase), TUBB4B (Tubulin beta-4B chain)). This cluster showed an overrepresentation of proteins involved in neoplasia and cancer transitions (Fig. 3Bd). Functional enrichment analyses point out differences between clusters. Different biological processes have been identified i.e. immune response, regulation of immune response, cell growth and/or maintenance, metabolism, energy pathways, signal transduction and protein metabolism (Fig. 4A). Enriched transcription factors (TFs) that regulate the overexpressed proteins in the different clusters were also obtained (Fig. 4B). The cluster containing proteins overexpressed in normal-p53 signature showed a high abundance of proteins involved in metabolism and energy pathways as well as an enrichment of SP1, SP4 and TEAD1 
transcription factors that targeted the most genes of this cluster. For the cluster normal-p53/STIL, proteins were mostly involved in cell growth and/or maintenance and protein metabolism. Enrichment of the TFs NFIC and EGR1 was observed. Concerning Normal-p53/STIC, identified proteins were implicated in cell growth and /or maintenance, metabolism, energy pathways and protein metabolism while KLF7 protein was identified as a TF. For STIL/STIC cluster, enrichment of the TFs ZEB1 and ETS1 was observed. STIC cluster presented a strong enrichment of proteins involved in protein metabolism and regulation of immune response and SP1, SP4 and KLF7 as TFs. We also observed a diminution of the proteins involved in immune response from normal-p53 to STIC. Cellular components analyses reflected that all proteins of the 5 clusters were in cytoplasm or exosomes (Fig. 4C) but with some differences. In fact, in STIL-STIC and STIC, we observed a strong enrichment for exosomes; whereas, p53 signature and STIC involved cytoplasmic proteins. We also observed a high enrichment of cytoskeletal proteins in STIC lesions. Altogether, these analyses reflectedsome clear transition occurring between Nomal-p53 signature, p53 to STIL and STIL to STIC with a modulation of proteins involved in cell growth and/or maintenance and the different metabolism processes. To confirm these observations, further analyses were carried out, this time grouping together all proteins overexpressed in each lesion, i.e., normal-p53 signature, STIL, and STIC (Fig. 4D, E and F and Supp. Data 3). In STIL, an enrichment of proteins involved in cell growth and/or maintenance (Fig. 4D), in integrin cell surface interactions (Fig. 4E), in extracellular matrix (ECM) structural constituent and cell adhesion molecule activity (Fig. 4F) were observed and the ones in various metabolism and energy pathways (Fig. 4D and E), catalytic activity (Fig. 4F) were lower than for the other cellular transition. We also investigated whether the proteins involved in the Warburg effect (Scatena et al., 2010) are activated to compensate for the overall decrease in the classical metabolism processes observed (Figure S1). These proteins are not altered overall with a decreasing rather than an increasing trend, which means that no Warburg effect seemed to occur. In STIC, proteins involved in metabolism, energy pathways, cell communication, signal transduction (Fig. 4D and E), calcium ion binding, catalytic and structural molecule activities (Fig. 4F) were higher than the other transition. In the same time, immune response (Fig. 4D), integrin interactions (Fig. 4E), cell adhesion molecule activity and ECM structural constituent (Fig. 4F) were highly repressed. Statistical overrepresentation test using PANTHER classification system analyses (Supp. Data 4) confirmed such transition.

Nevertheless, there are still difficulties in distinguishing between p53 signature and normal tissue. Using the Student's T-test, 34 proteins have been shown to be highly discriminant between Normal and p53 lesions with 26 overexpressed in Normal and 8 in p53 signature (Fig. 5, Supp. Data 5). Table 2 presents the immunoreactivity in normal FTE and prognostic effect on the overall survival (OS) in OC for these 8 proteins extracted from the HPA. PTRF, also named CAVIN1, is an unfavorable prognosis marker for ovarian, urothelial, and colorectal cancers, whereas SDPR (known as CAVIN2) is a renal cancer favorable prognosis marker and unfavorable in stomach cancer. The IHC data indicated that these proteins are not detected in normal FTE, showed a moderate positivity in OC for CAVIN1 and a strong positivity in a rare case of endometrioid carcinoma of ovary for CAVIN2. SPTAN1, FBLN5 are a favorable prognostic marker in renal cancer but unfavorable in OC (considering a $p<0.05$ ). EIF3B, MOB1B and Emilin2 are already known to bad prognostic markers for liver cancer. Moreover, EMILIN-2 and EIF3B are also considered as bad prognosis markers for renal cancer and for head neck cancer. 
Table 2

Immunoreactivity and prognostic effect of proteins overexpressed in p53 signature compared to normal tissue. Immunohistochemical staining of proteins in normal FTE tissue and prognostic effect of high expression of the corresponding gene in OVC were extracted from Human protein atlas (data credit: Human Protein Atlas available from v19.proteinatlas.org, $p$-value is represented by stars *** $p<0.001$, ** $p<0.01$, * $p<0.05$ and no star for $p>$ $0.05)$

\begin{tabular}{|lll|}
\hline Protein name & Immunoreactivity in FTE & Prognostic effect in OVC \\
\hline CAVIN1 & Not detected & Unfavorable *** \\
\hline SPTAN1 & Moderate to strong positivity & Unfavorable * \\
\hline FBLN5 & Not detected & Unfavorable * \\
\hline EIF3B & Strong positivity & Not significant \\
\hline MOB1B & Moderate positivity & Not significant \\
\hline EMILIN2 & Moderate positivity & Not significant \\
\hline SPTBN1 & Moderate to strong positivity & Not significant \\
\hline CAVIN2 & Not detected & Not significant \\
\hline
\end{tabular}

Considering the 26 proteins downregulated in the p53 signature, different pathways were down-represented in these samples, i.e., Neutrophil degranulation, SRP-dependant cotranslational protein targeting to membrane and cadherin binding function.

\section{Protein Mutation}

The lineage relationship between HGSC and the different lesions is suggested by the presence of same genomics changes and mutations (Kindelberger et al., 2007). In order to identify specific modifications that may have occurred during the different potential pre-cancerous lesions, we explored the presence of protein mutations per each lesion. For that purpose, we used the human database combined with the XMAn database (Flores and Lazar, 2020). This database contains information concerning mutated peptides that could be found in some cancers extracted from the COSMIC database. This database integration resulted in the identification of 83 peptide sequences containing possible mutations (Supp. Data 6). For four of them, it was possible to determine the amino acid modification directly via the tandem mass spectra (Fig. 6A). These peptides were derived from four proteins i.e., the vitamin D binding protein (GC), the polyubiquitin-C (UBC), the Histone H2B (H2B1C), and Histone H3.1 (H31). The mutations found in the protein GC (1296T > G p.D432E), lead the sequence LPEATPTELAK in the protein and has been identified in stomach cancer studies according to the COSMIC database (Alfaro et al., 2017; Dopazo and Erten, 2017; Gnad et al., 2013; Marquard et al., 2015). It was observed in data issued from two patients in a normal tissue and a p53 signature lesion. Interestingly, this protein was found to be significantly under-represented in the STIC lesion (Supp. Data 3). Similarly, the mutated peptide of the UBC was found in two normal tissues, two p53 signature lesions, and one STIL. The mutation (c.368G > C p.G123A), which leads to the new peptide the LIFAAKQLEDGR was previously identified in breast cancer. The mutated peptide (QVHPDTGISTK) from Histone H2B (mutation c.169T > A p.S57T) was identified only in one normal tissue while the protein was not dysregulated (Supp. Data 3). Interestingly, this mutation has been identified before in the endometrium. The peptide of the histone H3.1 (VTIMPRDIQLAR) was observed in two normal tissue, one p53 signature, and one STIL lesion. This 
has been found to be downregulated in the STIC lesion (Supp. Data 3). This mutation (c.368A > G p.K123R) has also been identified in the endometrium.

Considering the 83 mutations identified (Table 3, Sup. Data 6), some have been detected in normal to all lesion stages including the proteins: vimentin or collagen, but some are specific to one particular type e;g. mutations on Histone H4, Histone H2B type 1-C/E/F/G/I in p53 signature, mutation on 60S ribosomal protein L14 in STIL, and mutation on $(\mathrm{Na}(+) / \mathrm{H}(+)$ exchange regulatory cofactor NHE-RF1 in STIC. Interestingly, in the transition from Normal to $\mathrm{p} 53$ signature, 7 mutated peptides have been identified. For p53 signature/STIL and Normal-p53/STIL, only one mutation has been observed respectively on Isoform 2 of Tropomyosin beta chain and Histone H1.4 proteins. From normal- p53/STIL and STIC, 3 mutated peptides have been identified corresponding to Laminin subunit gamma 1, Vimentin, and Histone H3.1.It is note worthy that the last two mutations have been detected in the endometrium. 
Table 3

Mutation detected in proteins from proteomic data obtained by spatially resolved proteomic using XMAn database.

\begin{tabular}{|c|c|c|c|c|}
\hline Found in samples & $\begin{array}{l}\text { Patients* } \\
\text { Number }\end{array}$ & Protein & Mutation & Cancers \\
\hline \multirow[t]{2}{*}{ p53 signature } & 2 & Histone H4 & c.239A > Gp.K80R|AKRRTVT|Missense & Endometrium \\
\hline & 2 & $\begin{array}{l}\text { Histone H2B } \\
\text { type 1- } \\
\text { C/E/F/G/I }\end{array}$ & c.181G > T p.G61C|KAMCIMN|Missense & Endometrium \\
\hline STIL & 3 & $\begin{array}{l}60 S \text { ribosomal } \\
\text { protein } L 14\end{array}$ & $\begin{array}{l}\text { p.A159_K160insAA|AAAAAKVP|Insertion } \\
\text { - in frame }\end{array}$ & $\begin{array}{l}\text { Endometrium, } \\
\text { Ovary, Breast }\end{array}$ \\
\hline STIC & 2 & $\begin{array}{l}\mathrm{Na}(+) / \mathrm{H}(+) \\
\text { exchange } \\
\text { regulatory } \\
\text { cofactor NHE- } \\
\mathrm{RF} 1\end{array}$ & $\begin{array}{l}\text { c. } 767 \mathrm{C}>\mathrm{A} \\
\text { p.P256H|GPLHVPF|Missense| }\end{array}$ & $\begin{array}{l}\text { Large } \\
\text { intestine }\end{array}$ \\
\hline \multirow[t]{7}{*}{$\begin{array}{l}\text { Normal-p53 } \\
\text { signature }\end{array}$} & $2 / 2$ & $\begin{array}{l}\text { Hemoglobin } \\
\text { subunit delta }\end{array}$ & c.427G > T p.A143S|NALSHKY|Missense & Lung \\
\hline & $2 / 2$ & $\begin{array}{l}\text { Histone H2B } \\
\text { type } 1- \\
\text { C/E/F/G/I }\end{array}$ & c.133G > C p.V45L|VYKLLKQ|Missense & Breast \\
\hline & $\begin{array}{l}2 / 2 / 1 \\
\text { HGSC }\end{array}$ & $\begin{array}{l}\text { Actin, alpha } \\
\text { cardiac } \\
\text { muscle } 1\end{array}$ & c.166G > A p.V56||DSYIGDE|Missense & Endometrium \\
\hline & $1 / 3$ & $\begin{array}{l}\text { Synaptic } \\
\text { vesicle } \\
\text { membrane } \\
\text { protein VAT-1 } \\
\text { homolog }\end{array}$ & c.278T > G p.L93R|FADRMAR|Missense & Lung \\
\hline & $3 / 3$ & $\begin{array}{l}\text { Isoform } 2 \text { of } \\
\text { Drebrin-like } \\
\text { protein }\end{array}$ & $\begin{array}{l}\text { c. } 746 G \text { > A } \\
\text { p.R249Q|SRNQNEQ|Missense }\end{array}$ & Endometrium \\
\hline & $\begin{array}{l}2 / 3 / 1 \\
\text { STIL }\end{array}$ & $\begin{array}{l}\text { Isoform } 2 \text { of } \\
\text { Synaptopodin- } \\
2\end{array}$ & $\begin{array}{l}\text { c. } 3392 \mathrm{C}>\mathrm{T} \\
\text { p.P1131L|RPTLWEA|Missense }\end{array}$ & Skin \\
\hline & $\begin{array}{l}2 / 2 / 1 \\
\text { STIL }\end{array}$ & $\begin{array}{l}\text { polyubiquitin } \\
\mathrm{C}\end{array}$ & c.368G > C p.G123A|IFAAKQL|Missense & Breast \\
\hline p53signature/STIL & $2 / 3$ & $\begin{array}{l}\text { Isoform } 2 \text { of } \\
\text { Tropomyosin } \\
\text { beta chain }\end{array}$ & $\begin{array}{l}\text { c.835C > A } \\
\text { p.L279M|QTLMELN|Missense }\end{array}$ & $\begin{array}{l}\text { Autonomic } \\
\text { ganglia }\end{array}$ \\
\hline Normal-p53/STIL & $\begin{array}{l}2 / 3 / 2: 1 \\
\text { STIC }\end{array}$ & Histone H1.4 & c.262G > T p.V88L|KSLLSKG|Missense & Esophagus \\
\hline \multirow[t]{2}{*}{$\begin{array}{l}\text { Nomal- } \\
\text { p53/STIL/STIC }\end{array}$} & $\begin{array}{l}1 / 3 / 3 / 2 / 1 \\
\mathrm{HGSC}\end{array}$ & Vimentin & c. $1313 \mathrm{C}>$ G p.S438*|DTH|Nonsense & Endometrium \\
\hline & $4 / 4 / 3 / 2$ & $\begin{array}{l}\text { Laminin } \\
\text { subunit } \\
\text { gamma-1 }\end{array}$ & $\begin{array}{l}\text { c. } 2033 \mathrm{C}>\mathrm{T} \\
\text { p.P678L|SARLGPG|Missense }\end{array}$ & Breast \\
\hline
\end{tabular}

*Only mutations found in more than two patients 


\begin{tabular}{|cllll|}
\hline Found in samples & $\begin{array}{l}\text { Patients } \\
\text { Number }\end{array}$ & Protein & Mutation & Cancers \\
\hline $\begin{array}{l}\text { 4/4/4/4: Histone H3.1 } \\
\text { HGSC }\end{array}$ & c.368A > G p.K123R|IMPRDIQ|Missense & Endometrium \\
\hline *Only mutations found in more than two patients & & \\
\hline
\end{tabular}

\section{Ghost Proteome}

Using our proteomic data against the OpenProt database, 59 AltProts have been identified as ghost proteome. More than $64 \%$ of these proteins are associated with sequences from non-coding RNA (ncRNA), and from the mRNA coding for RefProt $23 \%$ are from 5'UTR, 33\% from the 3'UTR and $42 \%$ from a shift in the CDS (Supp. Data 7). Among these proteins (Table 4, Supp. Data 7), IP_270787 (AltZNF709) is the single specific accession of normal tissue. IP_2326985 is issued from an ncRNA (LOC105376003) and is only found in STIC. The AltProt IP_232994 (AltMYEF2) coded by the 3 'UTR part of the transcript mRNA referenced to translate the MYEF2 is identified only in HGSC samples. IP_572777 (from the ncRNA: SDR16C6P), IP_774783 (from the ncRNA: RP11-358N4.6) and IP_285024 (from the ncRNA: HNF4A) are specific of Normal, p53 signature and STIL samples. In both Normal/p53/STIL and STIC samples, 17 AltProts were identfied (Table 4). Using the ANOVA test, 12 AltProts present a significant variation in the different lesion stages. Nine AltProt are derived from ncRNA, one from $3^{\prime} U T R$, and two from a shift in the CDS. The hierarchical clustering and heatmap representation (Fig. 6Ba) revealed a clear separation between STIC and the other lesion. The second branch separates STIL from the normal-p53 signature. The pseudogene KRT8P11 (IP_563986) is overexpressed in STIC, whereas LOC105376924 and, COL18A1 (IP_290397) pseudogene were overexpressed in the STIL samples. The other AltProts are differentially expressed in p53 lesions and Normal tissue, but all are underexpressed in the STIC or non-expressed in STIL (Fig. 6Ba). When data from HGSC were added, the separation of STIC from other samples is still observed, carcinoma samples composed the second branch, and a third branch is divided into subbranches. These subdivisions group included three of the fourth STIL samples and three of the fourth Normal samples (Fig. 6Bb). With regards to the results, KRTP8P11 (IP_563986) is a specific marker of STIC, B4GALN (IP_191334) is specific of HGSC, and KRT8P32 (IP_602534) is overexpressed in two p53 signature samples and one of the normal tissue sample but also highly decreased in HGSC. Finaly, COL18A1 (IP_290397) is specific to STIL. The other AltProt were observed between Normal-p53 signatures with most of them under-expressed in STIC (Fig. 6Bb). 
Table 4

Summary of the repartition to the Venn diagram result of the 45

AltProts identified. 14 AltProt have been deleted because they are identified but without a sufficient abundance to be quantified. The AltProt identification accession ID is according to the OpenProt Database. Venn diagram representation is based on the abundance obtain after analysis, function to the triplicate samples type (normal, p53, STIL, STIC, Carcinoma).

\begin{tabular}{|c|c|c|c|c|c|}
\hline Altprot & Normal & P53 & STIL & STIC & Carcinoma \\
\hline IP_270787 & $x$ & & & & \\
\hline IP_572777 & $x$ & $x$ & $x$ & & \\
\hline IP_774783 & $x$ & $x$ & $x$ & & \\
\hline IP_285024 & $x$ & $x$ & $x$ & & \\
\hline IP_669028 & $x$ & $x$ & $x$ & $x$ & \\
\hline IP_563986 & $x$ & $x$ & $x$ & $x$ & \\
\hline IP_621829 & $x$ & $x$ & $x$ & $x$ & \\
\hline IP_595290 & $x$ & $x$ & $x$ & $x$ & \\
\hline IP_730500 & $x$ & $x$ & $x$ & $x$ & \\
\hline IP_2322038 & $x$ & $x$ & $x$ & $x$ & \\
\hline IP_763981 & $x$ & $x$ & $x$ & $x$ & \\
\hline IP_708166 & $x$ & $x$ & $x$ & $x$ & \\
\hline IP_737172 & $x$ & $x$ & $x$ & $x$ & \\
\hline IP_290397 & $x$ & $x$ & $x$ & $x$ & \\
\hline IP_652773 & $x$ & $x$ & $x$ & $x$ & \\
\hline IP_689518 & $x$ & $x$ & $x$ & $x$ & \\
\hline IP_721145 & $x$ & $x$ & $x$ & $x$ & \\
\hline IP_060976 & $x$ & $x$ & $x$ & $x$ & \\
\hline IP_632428 & $x$ & $x$ & $x$ & $x$ & \\
\hline IP_274301 & $x$ & $x$ & $x$ & $x$ & \\
\hline IP_138273 & $x$ & $x$ & $x$ & $x$ & \\
\hline IP_716829 & $x$ & & $x$ & $x$ & \\
\hline IP_2326985 & & & & $x$ & \\
\hline IP_591310 & & $x$ & $x$ & $x$ & $x$ \\
\hline IP_2285716 & $x$ & & $x$ & $x$ & $x$ \\
\hline IP_572435 & $x$ & $x$ & & $x$ & $x$ \\
\hline IP_2323408 & $x$ & & & $x$ & $x$ \\
\hline
\end{tabular}

Page $18 / 37$ 


\begin{tabular}{|c|c|c|c|c|c|}
\hline Altprot & Normal & P53 & STIL & STIC & Carcinoma \\
\hline IP_079312 & & $x$ & $x$ & & $x$ \\
\hline IP_667690 & & $x$ & $x$ & & $x$ \\
\hline IP_076499 & & $x$ & $x$ & & $x$ \\
\hline IP_601870 & $x$ & & $x$ & & $x$ \\
\hline IP_755940 & $x$ & $x$ & & & $x$ \\
\hline IP_232994 & & & & & $x$ \\
\hline IP_602534 & $x$ & $x$ & $x$ & $x$ & $x$ \\
\hline IP_667001 & $x$ & $x$ & $x$ & $x$ & $x$ \\
\hline IP_755869 & $x$ & $x$ & $x$ & $x$ & $x$ \\
\hline IP_658355 & $x$ & $x$ & $x$ & $x$ & $x$ \\
\hline IP_724315 & $x$ & $x$ & $x$ & $x$ & $x$ \\
\hline IP_591792 & $x$ & $x$ & $x$ & $x$ & $x$ \\
\hline IP_631742 & $x$ & $x$ & $x$ & $x$ & $x$ \\
\hline IP_688853 & $x$ & $x$ & $x$ & $x$ & $x$ \\
\hline IP_2371754 & $x$ & $x$ & $x$ & $x$ & $x$ \\
\hline IP_191334 & $x$ & $x$ & $x$ & $x$ & $x$ \\
\hline IP_773656 & $x$ & $x$ & $x$ & $x$ & $x$ \\
\hline IP_794359 & $x$ & $x$ & $x$ & $x$ & $x$ \\
\hline
\end{tabular}

\section{Discussion}

The most recent theories regarding the origin of HGSC propose an involvement of cells in the region of the tubalperitoneal junction (Leblanc et al., 2011; Lee et al., 2007; Mingels, 2015; Seidman et al., 2011). This junction corresponds to the region of the peritoneum covering the serosal surface of the fallopian tube and meets the specialized epithelium of the tubal fimbriae, with a transition between the Müllerian and the mesothelial cells (Gan et al., 2017). Such junctional sites between different types of epithelia represent cancer hot spots in which neoplastic transformation occurs. This is confirmed by evidence that cells derived from this transition region demonstrate a cancer-prone stem cell phenotype (Gan et al., 2017; Leblanc et al., 2011; Mingels, 2015; Seidman et al., 2011). Subsequent studies have led to the designation of these changes as STIC recognition of their preferential localization in the epithelium of the fimbriae end of the fallopian tube, and the recommendation of standardized and detailed sampling of tubal specimens with the SEE-FIM protocol. However, the diagnosis of STIC based on morphology alone is reported to have low reproducibility. With the emergence of detailed histological examination of the fallopian tubes, with and without IHC, a variety of confounding pre-neoplastic lesions is now being encountered which had not been previously characterized included p53 signature and STIL lesions (Carlson et al., 2010), but the temporal relationship between each lesion and HGSCs is still debated. 
The aim of our study was to investigate and the molecular mechanisms associated with the development of the pre-neoplastic lesions found in the FTE. To the best of our knowledge, no similar proteomics/bioinformatics studies have been condiucted yetinvestigating alterabtive and ghost proteomes, due to the the robust uniqueness of the protocol established for sampling the tissue sections. We firstly developed a novel strategy based on pathology routine protocol. Several tissue sections with pre-cancerous lesions are drastically limited. In fact, these types of lesions could only be observed using a SEE-FIM protocol as discussed above. It allows localization and differentiation of each lesion type but on a limited number of slides. For example, p53 signatures are observable only on one or two tissue sections. In this context, the direct use of the IHC slides was applied to perform other experiments has many advantages. In our study, to a better understanding of the different transitions of the lesions, i.e. from Normal to p53 signature, then from p53 signature to STIL and finally from STIL to STIC, IHC-guided spatially resolved proteomic analyses were realized on each identified lesion. We first demonstrated that the workflow we developed is compatible with proteomic analysis. Using targeted digestion of each region highlight by the IHC, we can preserve spatial information and perform analysis of different regions in the same slide. The tissue section surface recovery protocol does not alter the proteomic content and allows an accurate comparison of quantified proteins between each lesion.

If we compare the proteomic content of HGSC and the lesion found in the fallopian tube, we observed interesting groupings that may correspond to what different studies propose for the chronology of HGSC development from pre-neoplastic lesions. The normal tissue and the p53 signature cluster together and are close to the STIL. The STIC lesion presents a more different signature and is closer to HGSC than the two other lesions. Due to the difference in composition between the fallopian tube tissue and the ovary, we have focused our analysis to deepen our understanding of the biological processes involved in the different pre-neoplastic lesions.

By comparing the global content of each lesion, we successfully identified specific markers for p53 signature, the protein RNA-binding protein 10 (RBM10)- 3 specific proteins in STIC lesions -i.e., Glucosamine (N-acetyl)-6-sulfatase (GNS), Upstream binding transcription factor, RNA polymerase I (UBTF) and ATPase family, AAA domain containing 3 (ATAD3A/B). We also identified specific mutated proteins from each lesion stages i.e. mutated Histone $\mathrm{H} 4$, Histone H2B type 1-C/E/F/G/I) in p53 signature, mutated 60 S ribosomal protein L14 in STIL, and mutated $(\mathrm{Na}(+) / \mathrm{H}(+)$ exchange regulatory cofactor NHERF1 in STIC.

The p53 signature lesion is only characterized by a limited number of epithelial cells $(\sim 10)$ presenting a p53 overexpression. In our analyses, the p53 signature samples are close in their protein content to the normal tissue. But some proteins specific to these lesions could be obtained. A signature emerged and demonstrated that among the 8 proteins overrepresented in p53 signature, some of them are unfavorable prognosis markers of OC (CAVIN1, SPTAN1 and FBLN5) and some for renal, head and neck cancers (EIF3B) or liver cancer (MOB1B,EMILIN-2, EIF3B), by contrast, 3 are favorable prognosis markers for renal cancer (SPTAN1, SPTBN1, CAVIN2). We also dissected the molecular mechanisms that occur and the whole proteome analysis showed that the transition from normal to p53 signature was characterized by a residual immune response and translation activation, regulation of trafficking through cavins proteins, and ECM modifications through emilins.

From p53 signature to STIL: upregulated proteinswere involved in the adhesion and anchoring of cells in the ECM, allowing stabilization of the cells, along with an increase in maintenance activity instead of cell proliferation. Immune response and inflammation processes are still maintained, but an overall decrease of all metabolic processes, especially in TCA cycle is observed and with no Warburg effect occurs. These observations are in line with a recent study suggesting that STIL could be considered as "Dormant STICs" and take a prolonged time of

Page 20/37 
more than one decade to develop into STIC (Wu et al., 2019). We observed that mostly pathway concerning metabolism and cell growth and maintenance is downregulated in STIL that could explain this "dormant" characteristic.

From STIL to STIC: Contrary to the dormant profile of STIL lesion, observations done on the different function dysregulation for STIC lesion, suggest a more aggressive profile with a decrease of proteins involved in the cell adhesion and could reflect the start of an invasion step. Proteins that are upregulated in the STIC show various functions inside the cells and appear to be involved in different steps of cancer processes. An increase of proteins involved in the metabolism process and energy pathway are observed. If we compare the molecular functions involved in the three different groups, calcium-dependent protein binding is overrepresented. Also, heat shock protein activity is highly represented in this dataset. Proteins involved in telomere activities and centrosome maturation are specific to STICs compared to other lesions.

Interestingly, these two processes have been shown to be linked with early tumorigenesis (Kuhn et al., 2010) and that STIC precedes the development of many HGSCs (Kuhn et al., 2016). A decrease of expression of proteins involved in the extracellular structure organization and cell adhesion molecule activity was observed in STIC lesion, suggesting a breaching of the basement membrane and an increase of cell motility that could be conducted to an escape of these pre-cancerous cells to a distant organ. Finally, we observed a high level of proteins involved in extracellular vesicles including exosomes production which could consequently modify the microenvironment and promote cancerization processes.

From lesions to HGSC: One protein is found common in the pre-neoplastic lesions and HGSC, the Fibrillarin (FBL) protein. $\mathrm{FBL}$ is one of the core proteins of box C/D small nucleolar ribinucleoprotein complexes (snoRNP). This complex is involved in the first steps of pr-rRNA processing (Newton et al., 2003). An overexpression of FBL contributes to tumorigenesis and confers cellular resistance to chemo drugs (El Hassouni et al., 2020). It has also been shown that dysregulation of ribosome biogenesis plays key roles in oncogenesis (Truitt and Ruggero, 2016). Overactivation in cancer cells of ribosome biogenesis could be due to a loss of function of RNA polymerase repressors such as p53 (Zhai et al., 2000). Particularly FBL expression has been demonstrated to be correlated with p53 activity (Marcel et al., 2013). High levels of FBL protein were associated with the expression of mutant p53 and contributed to tumorigenesis by altering translational control of key cancer genes. UBTF is only present in STIC and HGSC. This protein is known to be a transcription factor involved in the regulation of the RNA polymerase I (Pol I), implicated in the regulation of cell cycle checkpoint and DNA damage response (Sanij et al., 2015)and regulated by MYC (Rossetti et al., 2018). different studies have shown a link between Pol I initiation factor and chemoresistance of ovarian cancer (Cornelison et al., 2017)

Similarities between these pre-neoplastic lesions and HGSC have been found especially concerning TP53 mutations and other genomic changes, but without excessive cell proliferation (Yamamoto et al., 2016). In that context, we decided to explore the presence of mutations at the protein level in the different steps of tubal tumor development. Using a specific database for protein mutation, XMAn, resulted in the identification of 83 mutated peptides sequences, of which 4 were observed directly in the tandem mass spectra. Taken together, it is interesting to observe that most of the protein mutated in the p53 signature have been initially identified in endometrium cancer and are related to Histones and ribosomes suggesting a link with the epigenetic and translation.

Polyubiquitin $\mathrm{C}$ is related to proteasome and self-antigen presentation. Synaptopodin-2 and the Isoform 2 of Drebrin-like protein are actin-binding proteins known to be respectively an invasive cancer biomarker (Kai et al., 
2015) and a potential marker for breast, lung, and colorectal cancers (Chen et al., 2016). Finally, the Actin, alpha cardiac muscle 1 is involved in cisplatin ovarian cancer cell resistance (Pan et al., 2009) and the synaptic vesicle membrane protein VAT-1 homolog is a marker of epithelial cells (Koch et al., 2003) and an oncogene in gastric cancer (Mottaghi-Dastjerdi et al., 2016). None of these mutated proteins have been found in STIL and STIC except the ones occurring on histones. Most of the mutated peptides observed in STIL, STIC, p53/STIL, or in Normal/p53 signature/STIL/STIC are linked to the cytoskeleton and migration. These mutations could be key drivers of the cancer process from the epigenetic modification step, and then on cytoskeleton modifications for cell migration and cancer development.

Using a dedicated database, identification of significantly variable AltProts, provide us with a new view of unknown markers. We previously established the presence of cancer-promoting proteins from non-canonical human open reading frames (AltORF) in HGSC and glioma,(Cardon et al., 2020; Cardon et al., 2019; Delcourt et al., 2018; Vanderperre et al., 2013; Vergara et al., 2020). Among the alternative proteins (AltProt) observed in ovarian serous cancer, we already identified AltEDARADD, which is now annotated by Uniprot with the accession number L0R849. Interestingly, a high expression level of AltEDARADD protein is associated with a poor prognosis for patients with ovarian HGSC (Cardon et al., 2020). Using Top-down proteomic strategy, we identified six AltProt specific to the benign region (AltTLR5, AltPKHD1L1, AtltSERPINE1, AltGPC5, AltGRAMD4, AltAGAP1), five in the necrotic/fibrotic tumor region (AltApol6, AltLARS2-AS1, AltLTB4R, AltTMP1, AltMTHFR) and four in the tumor (AltCMBL, AltGNL1, AltRP11-576E20.1, AltCSNK1A1L) (Delcourt et al., 2017). AltGNL1 was selected for further analysis. In these conditions, AltGNL1 displayed a nuclear localization, whereas GNL1 was present in the cytosol (Delcourt et al., 2017).

In our present study, some of the identified AltProt or transcripts have been described in other cancer studies. The pseudogene KRT8P32 has been identified in breast cancer (Hardman et al., 2019), the pseudogene ACTBP11 is observed in GM12878 (B cells) cell line according to Diana-LncBase V3 (Karagkouni et al., 2020). Concerning the ANKRD28, this AltProt has been recently demonstrated to act as a novel BRCA1-interacting protein in breast and ovarian cancer (Vincent et al., 2016). RP11-13K12.2 is described to be overexpressed in pediatric ovarian fibrosarcoma (Melendez-Zajgla et al., 2018). COL18A1 and B4GALNT4 are overexpressed in endometrial and ovarian cancers according to the HPA and are unfavorable markers for overall survival for both cancers. Some of these are specific to the type of lesion - i.e. LOC105376924 and COL18A1 in STILL, KRT8P11 in STIC and ANKRD28 in STILL-STIC. These alternative proteins seem to be clinically relevant, and further exploration will lead to a better understanding of the disease and new targets to improve diagnosis.

Finally, the Identification of the AltProt and the high number of mutations affecting histones and cytoskeleton proteins observed between normal to p53 signature compared to the other lesions, are in line with the hypothesis of epigenetic reprogramming towards carcinogenesis and cell transformation (Bartlett et al., 2016). A recent study established that epigenetic reprogramming occurs specifically in the proximal end of the fallopian tubes in $B R C A$ mutation carriers. This epigenetic reprogramming event is driven by aberrantly high AICDA (also named AID, activation-induced cytosine deaminase) expression and is an integral early pre-malignant event in HGSC development. In this context, our results would provide an interesting starting point for further studies, in respect to the potential link to endometrium and exfoliation of endometrial carcinoma cells due to epigenetic reprogramming to carcinogenesis (Mingels et al., 2014) or that STIL represents exfoliated precursor cells that eventually undergo malignant transformation within the peritoneal cavity (Kuhn et al., 2012).

\section{Conclusion}

Page 22/37 
Overall, the present study identified several processes involved in the various neoplastic lesions that are observed in the fimbriated end of the fallopian tube. Besides, we have demonstrated the association across the different lesions, allowing us to identify the potential timeline and underly mechanisms of origin of ovarian cancer. Several of the identified protein hits represent potential markers for differentiating several classes of ovarian related cancers which require further validation in bigger cohorts of clinical samples.

\section{Abbreviations}

FTE fallopian tube epithelium

EMT Epithelial-mesenchymal transition

FFPE formalin fixed paraffin embedded

GO Gene Ontology

HGSC High Grade Serous Carcinoma

HPA Human Protein Atlas

IHC immunohistochemistry

MALDI Matrix assisted Laser Desorption Ionization

MSI Mass spectrometry imaging

OC Ovarian cancer

OS overall survival

PCA principal component analysis

SEE-FIM Sectioning and Extensively Examining the Fimbriated End Protocol

STIC serous tubal intraepithelial carcinoma

STIL serous tubal intraepithelial lesions

TF Transcription factor

\section{Declarations}

Ethics approval and consent to participate : All experiments were approved by the local Ethics Committee (CPP Nord Ouest IV 12/10) in accordance with the French and European legislation. Prior to the experiments, patients signed an informed consent and authorization form describing the experimental protocol. No personal information was used in these experiments, and a random number was assigned to each sample.

Consent for publication : All patient consent for publication 
Availability of data and material : The MS data sets and Perseus result files used for analysis were deposited at the ProteomeXchange Consortium (Vizcaino et al., 2014) (http://proteomecentral.proteomexchange.org) via the PRIDE partner repository (Vizcaino et al., 2014) with the data set identifier PXD020024 (for reviewer access only, Username: reviewer86596@ebi.ac.uk; Password: x88jqaDV).

Competing interest : The authors declare no competing financial interests in this work.

This research was supported by grants from the Ministère de L'Education Nationale, de L'Enseignement Supérieur et de la Recherche, ANR (IF), SIRIC ONCOLille (IF), Grant INCa-DGOS-Inserm 6041aa, and INSERM, the PHRC FIMBRIA (EL), Ligue contre le Cancer (MW).

Author contributions: IF, MW and MS conceived and supervised the study. MW, PS performed the experiments. Surgery was realized by EL and FN. The pathology analyses were done by YMR DB, ASL. FK, SA, MW performed the systemic biology analyses. IF, EL, MW, PS and MS participated in experimental design. MW, SA, and PS performed data analyses. MS and MW wrote the manuscript with contributions from all co-authors. MS, EL, MW, and IF found funds for the project.

Acknowledgements : The authors thanks Annie Desmons for her technical help in this project.

\section{References}

Alfaro, J. A., Ignatchenko, A., Ignatchenko, V., Sinha, A., Boutros, P. C., and Kislinger, T. (2017). Detecting protein variants by mass spectrometry: a comprehensive study in cancer cell-lines. Genome Med 9, 62.

Auersperg, N. J. F. B. (2013). The origin of ovarian cancers-hypotheses and controversies. 5, 709-719.

Bachert, S. E., McDowell, A., Piecoro, D., and Baldwin Branch, L. J. D. (2020). Serous Tubal Intraepithelial Carcinoma: A Concise Review for the Practicing Pathologist and Clinician. 10, 102.

Bartlett, T. E., Chindera, K., McDermott, J., Breeze, C. E., Cooke, W. R., Jones, A., Reisel, D., Karegodar, S. T., Arora, R., and Beck, S. J. N. c. (2016). Epigenetic reprogramming of fallopian tube fimbriae in BRCA mutation carriers defines early ovarian cancer evolution. 7, 1-10.

Bayarkhangai, B., Noureldin, S., Yu, L., Zhao, N., Gu, Y., Xu, H., and Guo, C. J. C. m. (2018). A comprehensive and perspective view of oncoprotein SET in cancer. 7, 3084-3094.

Bonnet, A., Lagarrigue, S., Liaubet, L., Robert-Granie, C., Sancristobal, M., and Tosser-Klopp, G. (2009). Pathway results from the chicken data set using GOTM, Pathway Studio and Ingenuity softwares. BMC Proc 3 Supp/ 4, S11.

Bowtell, D. D. N. R. C. (2010). The genesis and evolution of high-grade serous ovarian cancer. $10,803-808$.

Braicu, E., Sehouli, J., Richter, R., Pietzner, K., Denkert, C., and Fotopoulou, C. J. B. j. o. c. (2011). Role of histological type on surgical outcome and survival following radical primary tumour debulking of epithelial ovarian, fallopian tube and peritoneal cancers. 105, 1818-1824.

Brunet, M. A., Brunelle, M., Lucier, J. F., Delcourt, V., Levesque, M., Grenier, F., Samandi, S., Leblanc, S., Aguilar, J. D., Dufour, P., et al. (2019). OpenProt: a more comprehensive guide to explore eukaryotic coding potential and proteomes. Nucleic acids research 47, D403-D410.

Page 24/37 
Cardon, T., Herve, F., Delcourt, V., Roucou, X., Salzet, M., Franck, J., and Fournier, I. (2020). Optimized Sample Preparation Workflow for Improved Identification of Ghost Proteins. Anal Chem 92, 1122-1129.

Cardon, T., Salzet, M., Franck, J., and Fournier, I. (2019). Nuclei of HeLa cells interactomes unravel a network of ghost proteins involved in proteins translation. Biochim Biophys Acta Gen Subj 1863, 1458-1470.

Carlson, J. W., Jarboe, E. A., Kindelberger, D., Nucci, M. R., Hirsch, M. S., and Crum, C. P. J. I. j. o. g. p. (2010). Serous tubal intraepithelial carcinoma: diagnostic reproducibility and its implications. 29, 310-314.

Chen, W., Liu, G., Jin, M., Ju, P., Xu, J., Zhang, Y., and Zhang, H. J. I. J. C. E. M. (2016). Drebrin is a potential diagnostic biomarker for breast cancer. 9, 23598-23604.

Cornelison, R., Dobbin, Z. C., Katre, A. A., Jeong, D. H., Zhang, Y., Chen, D., Petrova, Y., Llaneza, D. C., Steg, A. D., and Parsons, L. (2017). Targeting RNA-polymerase I in both chemosensitive and chemoresistant populations in epithelial ovarian cancer. Clinical Cancer Research 23, 6529-6540.

Cox, J., Hein, M. Y., Luber, C. A., Paron, I., Nagaraj, N., and Mann, M. (2014). Accurate proteome-wide label-free quantification by delayed normalization and maximal peptide ratio extraction, termed MaxLFQ. Molecular \& cellular proteomics : MCP 13, 2513-2526.

Cox, J., and Mann, M. (2008). MaxQuant enables high peptide identification rates, individualized p.p.b.-range mass accuracies and proteome-wide protein quantification. Nat Biotechnol 26, 1367-1372.

Creekmore, A. L., Silkworth, W. T., Cimini, D., Jensen, R. V., Roberts, P. C., and Schmelz, E. M. J. P. o. (2011). Changes in gene expression and cellular architecture in an ovarian cancer progression model. 6 .

Crum, C. P., Drapkin, R., Kindelberger, D., Medeiros, F., Miron, A., Lee, Y. J. C. m., and research (2007). Lessons from BRCA: the tubal fimbria emerges as an origin for pelvic serous cancer. 5, 35-44.

Delcourt, V., Brunelle, M., Roy, A. V., Jacques, J. F., Salzet, M., Fournier, I., and Roucou, X. (2018). The protein coded by a short open reading frame, not by the annotated coding sequence is the main gene product of the dual-coding gene MIEF1. Molecular \& cellular proteomics : MCP.

Delcourt, V., Franck, J., Leblanc, E., Narducci, F., Robin, Y. M., Gimeno, J. P., Quanico, J., Wisztorski, M., Kobeissy, F., Jacques, J. F., et al. (2017). Combined Mass Spectrometry Imaging and Top-down Microproteomics Reveals Evidence of a Hidden Proteome in Ovarian Cancer. EBioMedicine 21, 55-64.

Dopazo, J., and Erten, C. (2017). Graph-theoretical comparison of normal and tumor networks in identifying BRCA genes. BMC Syst Biol 11, 110.

El Hassouni, B., Granchi, C., Vallés-Martí, A., Supadmanaba, I. G. P., Bononi, G., Tuccinardi, T., Funel, N., Jimenez, C. R., Peters, G. J., and Giovannetti, E. (2020). The dichotomous role of the glycolytic metabolism pathway in cancer metastasis: interplay with the complex tumor microenvironment and novel therapeutic strategies. Paper presented at: Seminars in Cancer Biology (Elsevier).

Flores, M. A., and Lazar, I. M. J. B. (2020). XMAn v2-a database of Homo sapiens mutated peptides. 36, 13111313. 
Folkins, A. K., Jarboe, E. A., Roh, M. H., and Crum, C. P. J. G. O. (2009). Precursors to pelvic serous carcinoma and their clinical implications. 113, 391-396.

Francavilla, C., Lupia, M., Tsafou, K., Villa, A., Kowalczyk, K., Jersie-Christensen, R. R., Bertalot, G., Confalonieri, S., Brunak, S., and Jensen, L. J. J. C. r. (2017). Phosphoproteomics of primary cells reveals druggable kinase signatures in ovarian cancer. $18,3242-3256$.

Gan, C., Chenoy, R., Chandrasekaran, D., Brockbank, E., Hollingworth, A., Vimplis, S., Lawrence, A. C., Jeyarajah, A. R., Oram, D., Deo, N. J. A. j. o. o., and gynecology (2017). Persistence of fimbrial tissue on the ovarian surface after salpingectomy. 217, 425. e421-425. e416.

Gnad, F., Baucom, A., Mukhyala, K., Manning, G., and Zhang, Z. (2013). Assessment of computational methods for predicting the effects of missense mutations in human cancers. BMC Genomics 14 Supp/ 3, S7.

Hardman, W. E., Primerano, D. A., Legenza, M. T., Morgan, J., Fan, J., and Denvir, J. J. D. i. b. (2019). mRNA expression data in breast cancers before and after consumption of walnut by women. 25, 104050 .

Kahlert, C., Weber, H., Mogler, C., Bergmann, F., Schirmacher, P., Kenngott, H., Matterne, U., Mollberg, N., Rahbari, N., and Hinz, U. J. B. j. o. c. (2009). Increased expression of ALCAM/CD166 in pancreatic cancer is an independent prognostic marker for poor survival and early tumour relapse. 101, 457-464.

Kai, F., Fawcett, J. P., and Duncan, R. J. O. (2015). Synaptopodin-2 induces assembly of peripheral actin bundles and immature focal adhesions to promote lamellipodia formation and prostate cancer cell migration. 6,11162 .

Kalra, R. S., and Bapat, S. A. J. P. o. (2013). Expression proteomics predicts loss of RXR-y during progression of epithelial ovarian cancer. 8 .

Karagkouni, D., Paraskevopoulou, M. D., Tastsoglou, S., Skoufos, G., Karavangeli, A., Pierros, V., Zacharopoulou, E., and Hatzigeorgiou, A. G. J. N. a. r. (2020). DIANA-LncBase v3: indexing experimentally supported miRNA targets on non-coding transcripts. 48, D101-D110.

Kindelberger, D. W., Lee, Y., Miron, A., Hirsch, M. S., Feltmate, C., Medeiros, F., Callahan, M. J., Garner, E. O., Gordon, R. W., and Birch, C. J. T. A. j. o. s. p. (2007). Intraepithelial carcinoma of the fimbria and pelvic serous carcinoma: evidence for a causal relationship. 31, 161-169.

Kobayashi, H., Iwai, K., Niiro, E., Morioka, S., Yamada, Y., Ogawa, K., and Kawahara, N. J. B. r. (2017). The conceptual advances of carcinogenic sequence model in high-grade serous ovarian cancer. 7, 209-213.

Koch, J., Foekens, J., Timmermans, M., Fink, W., Wirzbach, A., Kramer, M. D., and Schaefer, B. M. J. A. o. d. r. (2003). Human VAT-1: a calcium-regulated activation marker of human epithelial cells. 295, 203-210.

Kuhn, E., Kurman, R. J., Vang, R., Sehdev, A. S., Han, G., Soslow, R., Wang, T. L., and Shih, I. M. (2012). TP53 mutations in serous tubal intraepithelial carcinoma and concurrent pelvic high-grade serous carcinoma-evidence supporting the clonal relationship of the two lesions. The Journal of pathology 226, 421-426.

Kuhn, E., Meeker, A., Wang, T.-L., Sehdev, A. S., Kurman, R. J., and Shih, I.-M. J. T. A. j. o. s. p. (2010). Shortened telomeres in serous tubal intraepithelial carcinoma: an early event in ovarian high-grade serous carcinogenesis. 34, 829. 
Kuhn, E., Wang, T.-L., Doberstein, K., Bahadirli-Talbott, A., Ayhan, A., Sehdev, A. S., Drapkin, R., Kurman, R. J., and Shih, I.-M. J. M. P. (2016). CCNE1 amplification and centrosome number abnormality in serous tubal intraepithelial carcinoma: further evidence supporting its role as a precursor of ovarian high-grade serous carcinoma. 29, 12541261.

Le Rhun, E., Duhamel, M., Wisztorski, M., Zairi, F., Maurage, C. A., Fournier, I., Reyns, N., and Salzet, M. (2015). METB07 Classification of high grade glioma using matrix-assisted laser desorption/ionization mass spectrometry imaging (MALDI MSI): interim results of the GLIOMIC study. Neuro-Oncology 17, v136-v136.

Leblanc, E., Narducci, F., Farre, I., Peyrat, J.-P., Taieb, S., Adenis, C., and Vennin, P. J. G. o. (2011). Radical fimbriectomy: a reasonable temporary risk-reducing surgery for selected women with a germ line mutation of BRCA 1 or 2 genes? Rationale and preliminary development. 121, 472-476.

Lee, Y., Miron, A., Drapkin, R., Nucci, M., Medeiros, F., Saleemuddin, A., Garber, J., Birch, C., Mou, H., Gordon, R. J. T. J. o. P. A. J. o. t. P. S. o. G. B., and Ireland (2007). A candidate precursor to serous carcinoma that originates in the distal fallopian tube. 211, 26-35.

Levin, T. G., Powell, A. E., Davies, P. S., Silk, A. D., Dismuke, A. D., Anderson, E. C., Swain, J. R., and Wong, M. H. J. G. (2010). Characterization of the intestinal cancer stem cell marker CD166 in the human and mouse gastrointestinal tract. $139,2072-2082$. e2075.

Li, J., Zuo, X., Shi, J., Zhang, J., Duan, X., and Xu, G. J. N. (2018). Flotillin 1 is differentially expressed in human epithelial ovarian tumors. $65,561-571$.

Liang, W., Gao, R., Yang, M., Wang, X., Cheng, K., Shi, X., He, C., Li, Y., Wu, Y., and Shi, L. J. O. L. (2020). MARCKSL1 promotes the proliferation, migration and invasion of lung adenocarcinoma cells. 19, 2272-2280.

Lunter, P. C., Van Kilsdonk, J. W., Van Beek, H., Cornelissen, I. M., Bergers, M., Willems, P. H., van Muijen, G. N., and Swart, G. W. J. C. R. (2005). Activated leukocyte cell adhesion molecule (ALCAM/CD166/MEMD), a novel actor in invasive growth, controls matrix metalloproteinase activity. 65, 8801-8808.

Marcel, V., Ghayad, S. E., Belin, S., Therizols, G., Morel, A.-P., Solano-Gonzàlez, E., Vendrell, J. A., Hacot, S., Mertani, H. C., and Albaret, M. A. J. C. c. (2013). p53 acts as a safeguard of translational control by regulating fibrillarin and rRNA methylation in cancer. $24,318-330$.

Marquard, A. M., Birkbak, N. J., Thomas, C. E., Favero, F., Krzystanek, M., Lefebvre, C., Ferte, C., Jamal-Hanjani, M., Wilson, G. A., Shafi, S., et al. (2015). TumorTracer: a method to identify the tissue of origin from the somatic mutations of a tumor specimen. BMC Med Genomics 8, 58.

Medeiros, F., Muto, M. G., Lee, Y., Elvin, J. A., Callahan, M. J., Feltmate, C., Garber, J. E., Cramer, D. W., and Crum, C. P. (2006a). The tubal fimbria is a preferred site for early adenocarcinoma in women with familial ovarian cancer syndrome. Am J Surg Pathol 30, 230-236.

Medeiros, F., Muto, M. G., Lee, Y., Elvin, J. A., Callahan, M. J., Feltmate, C., Garber, J. E., Cramer, D. W., and Crum, C. P. J. T. A. j. o. s. p. (2006b). The tubal fimbria is a preferred site for early adenocarcinoma in women with familial ovarian cancer syndrome. 30, 230-236. 
Melendez-Zajgla, J., Mercado-Celis, G. E., Gaytan-Cervantes, J., Torres, A., Gabiño, N. B., Zapata-Tarres, M., JuarezVillegas, L. E., Lezama, P., Maldonado, V., and Ruiz-Monroy, K. J. S. r. (2018). Genomics of a pediatric ovarian fibrosarcoma. Association with the DICER1 syndrome. 8, 1-12.

Mingels, M. (2015) Premalignancies of serous ovarian cancer in Müllerian derived epithelium, [SI: sn].

Mingels, M. J., Van Ham, M. A., De Kievit, I. M., Snijders, M. P., Van Tilborg, A. A., Bulten, J., and Massuger, L. F. J. M. P. (2014). Müllerian precursor lesions in serous ovarian cancer patients: using the SEE-Fim and SEE-End protocol. $27,1002-1013$.

Mottaghi-Dastjerdi, N., Soltany-Rezaee-Rad, M., Sepehrizadeh, Z., Roshandel, G., Ebrahimifard, F., and Setayesh, N. (2016). Gene expression profiling revealed overexpression of vesicle amine transport protein-1 (VAT-1) as a potential oncogene in gastric cancer.

Newton, K., Petfalski, E., Tollervey, D., Cáceres, J. F. J. M., and biology, c. (2003). Fibrillarin is essential for early development and required for accumulation of an intron-encoded small nucleolar RNA in the mouse. 23, 85198527.

Ofori-Acquah, S. F., and King, J. A. J. T. R. (2008). Activated leukocyte cell adhesion molecule: a new paradox in cancer. $151,122-128$.

Pal, T., Permuth-Wey, J., Betts, J. A., Krischer, J. P., Fiorica, J., Arango, H., LaPolla, J., Hoffman, M., Martino, M. A., and Wakeley, K. J. C. I. I. J. o. t. A. C. S. (2005). BRCA1 and BRCA2 mutations account for a large proportion of ovarian carcinoma cases. $104,2807-2816$.

Pan, S., Cheng, L., White, J. T., Lu, W., Utleg, A. G., Yan, X., Urban, N. D., Drescher, C. W., Hood, L., and Lin, B. J. O. A. J. o. I. B. (2009). Quantitative proteomics analysis integrated with microarray data reveals that extracellular matrix proteins, catenins, and p53 binding protein 1 are important for chemotherapy response in ovarian cancers. 13,345354.

Pathan, M., Keerthikumar, S., Ang, C. S., Gangoda, L., Quek, C. Y., Williamson, N. A., Mouradov, D., Sieber, O. M., Simpson, R. J., and Salim, A. J. P. (2015). FunRich: An open access standalone functional enrichment and interaction network analysis tool. 15, 2597-2601.

Peluso, J. J. (2011). Progesterone signaling mediated through progesterone receptor membrane component-1 in ovarian cells with special emphasis on ovarian cancer. 76, 903-909.

Quanico, J., Franck, J., Cardon, T., Leblanc, E., Wisztorski, M., Salzet, M., and Fournier, I. (2016). NanoLC-MS coupling of liquid microjunction microextraction for on-tissue proteomic analysis. Biochim Biophys Acta.

Quanico, J., Franck, J., Dauly, C., Strupat, K., Dupuy, J., Day, R., Salzet, M., Fournier, I., and Wisztorski, M. (2013). Development of liquid microjunction extraction strategy for improving protein identification from tissue sections. Journal of proteomics $79,200-218$.

Roh, M. H., Yassin, Y., Miron, A., Mehra, K. K., Mehrad, M., Monte, N. M., Mutter, G. L., Nucci, M. R., Ning, G., and Mckeon, F. D. J. M. P. (2010). High-grade fimbrial-ovarian carcinomas are unified by altered p53, PTEN and PAX2 expression. 23, 1316-1324. 
Rossetti, S., Wierzbicki, A. J., and Sacchi, N. (2018). Undermining ribosomal RNA transcription in both the nucleolus and mitochondrion: an offbeat approach to target MYC-driven cancer. Oncotarget 9, 5016.

Sanij, E., Diesch, J., Lesmana, A., Poortinga, G., Hein, N., Lidgerwood, G., Cameron, D. P., Ellul, J., Goodall, G. J., and Wong, L. H. (2015). A novel role for the Pol I transcription factor UBTF in maintaining genome stability through the regulation of highly transcribed Pol II genes. Genome research 25, 201-212.

Santin, A. D., Zhan, F., Bellone, S., Palmieri, M., Cane, S., Bignotti, E., Anfossi, S., Gokden, M., Dunn, D., and Roman, J. J. J. I. j. o. c. (2004). Gene expression profiles in primary ovarian serous papillary tumors and normal ovarian epithelium: identification of candidate molecular markers for ovarian cancer diagnosis and therapy. 112, 14-25.

Scatena, R., Bottoni, P., Pontoglio, A., and Giardina, B. J. P. C. A. (2010). Revisiting the Warburg effect in cancer cells with proteomics. The emergence of new approaches to diagnosis, prognosis and therapy. 4, 143-158.

Seidman, J. D., Horkayne-Szakaly, I., Haiba, M., Boice, C. R., Kurman, R. J., and Ronnett, B. M. J. I. j. o. g. p. (2004). The histologic type and stage distribution of ovarian carcinomas of surface epithelial origin. 23, 41-44.

Seidman, J. D., Yemelyanova, A., Zaino, R. J., and Kurman, R. J. J. I. j. o. g. p. (2011). The fallopian tube-peritoneal junction: a potential site of carcinogenesis. 30,4-11.

Shimamura, M., Nagayama, Y., Matsuse, M., Yamashita, S., and Mitsutake, N. J. E. j. (2014). Analysis of multiple markers for cancer stem-like cells in human thyroid carcinoma cell lines. 61,481-490.

Singh, N., Gilks, C. B., Wilkinson, N., and McCluggage, W. G. J. P. (2015). The secondary Müllerian system, field effect, BRCA, and tubal fimbria: our evolving understanding of the origin of tubo-ovarian high-grade serous carcinoma and why assignment of primary site matters. $47,423-431$.

Truitt, M. L., and Ruggero, D. J. N. R. C. (2016). New frontiers in translational control of the cancer genome. $16,288$. Tyanova, S., and Cox, J. (2018). Perseus: a bioinformatics platform for integrative analysis of proteomics data in cancer research. In Cancer systems biology, (Springer), pp. 133-148.

Tyanova, S., Temu, T., Carlson, A., Sinitcyn, P., Mann, M., and Cox, J. (2015). Visualization of LC-MS/MS proteomics data in MaxQuant. Proteomics 15, 1453-1456.

Uhlen, M., Zhang, C., Lee, S., Sjöstedt, E., Fagerberg, L., Bidkhori, G., Benfeitas, R., Arif, M., Liu, Z., and Edfors, F. J. S. (2017). A pathology atlas of the human cancer transcriptome. 357, eaan2507.

Vanderperre, B., Lucier, J. F., Bissonnette, C., Motard, J., Tremblay, G., Vanderperre, S., Wisztorski, M., Salzet, M., Boisvert, F. M., and Roucou, X. (2013). Direct detection of alternative open reading frames translation products in human significantly expands the proteome. PLoS One 8, e70698.

Vang, R., Visvanathan, K., Gross, A., Maambo, E., Gupta, M., Kuhn, E., Li, R. F., Ronnett, B. M., Seidman, J. D., and Yemelyanova, A. J. I. j. o. g. p. (2012). Validation of an algorithm for the diagnosis of serous tubal intraepithelial carcinoma. 31, 243.

Vergara, D., Verri, T., Damato, M., Trerotola, M., Simeone, P., Franck, J., Fournier, I., Salzet, M., and Maffia, M. (2020). A Hidden Human Proteome Signature Characterizes the Epithelial Mesenchymal Transition Program. Curr Pharm Des 
Vincent, A., Berthel, E., Dacheux, E., Magnard, C., and Venezia, N. L. D. J. B. J. (2016). BRCA1 affects protein phosphatase 6 signalling through its interaction with ANKRD28. 473, 949-960.

Visvanathan, K., Vang, R., Shaw, P., Gross, A., Soslow, R., Parkash, V., Shih, I.-M., and Kurman, R. J. J. T. A. j. o. s. p. (2011a). Diagnosis of serous tubal intraepithelial carcinoma based on morphologic and immunohistochemical features: a reproducibility study. $35,1766$.

Visvanathan, K., Vang, R., Shaw, P., Gross, A., Soslow, R., Parkash, V., Shih le, M., and Kurman, R. J. (2011b). Diagnosis of serous tubal intraepithelial carcinoma based on morphologic and immunohistochemical features: a reproducibility study. Am J Surg Pathol 35, 1766-1775.

Vizcaino, J. A., Deutsch, E. W., Wang, R., Csordas, A., Reisinger, F., Rios, D., Dianes, J. A., Sun, Z., Farrah, T., Bandeira, N., et al. (2014). ProteomeXchange provides globally coordinated proteomics data submission and dissemination. Nat Biotechnol 32, 223-226.

Wisztorski, M., Desmons, A., Quanico, J., Fatou, B., Gimeno, J. P., Franck, J., Salzet, M., and Fournier, I. (2016). Spatially-resolved protein surface microsampling from tissue sections using liquid extraction surface analysis. Proteomics $16,1622-1632$.

Wisztorski, M., Fatou, B., Franck, J., Desmons, A., Farre, I., Leblanc, E., Fournier, I., and Salzet, M. (2013). Microproteomics by liquid extraction surface analysis: application to FFPE tissue to study the fimbria region of tubo-ovarian cancer. Proteomics Clin Appl 7, 234-240.

Wisztorski, M., Franck, J., Salzet, M., and Fournier, I. (2010). MALDI direct analysis and imaging of frozen versus FFPE tissues: what strategy for which sample? Methods Mol Biol 656, 303-322.

Wisztorski, M., Quanico, J., Franck, J., Fatou, B., Salzet, M., and Fournier, I. (2017). Droplet-Based Liquid Extraction for Spatially-Resolved Microproteomics Analysis of Tissue Sections. Methods Mol Biol 1618, 49-63.

Wu, R. C., Wang, P., Lin, S. F., Zhang, M., Song, Q., Chu, T., Wang, B. G., Kurman, R. J., Vang, R., and Kinzler, K. J. T. J. o. p. (2019). Genomic landscape and evolutionary trajectories of ovarian cancer precursor lesions. 248, 41-50.

Yamamoto, Y., Ning, G., Howitt, B. E., Mehra, K., Wu, L., Wang, X., Hong, Y., Kern, F., Wei, T. S., and Zhang, T. J. T. J. o. p. (2016). In vitro and in vivo correlates of physiological and neoplastic human Fallopian tube stem cells. 238, 519530.

Yang, X., and Lazar, I. M. (2014). XMAn: a Homo sapiens mutated-peptide database for the MS analysis of cancerous cell states. Journal of proteome research 13, 5486-5495.

Yuryev, A., Kotelnikova, E., and Daraselia, N. (2009). Ariadne's ChemEffect and Pathway Studio knowledge base. Expert Opin Drug Discov 4, 1307-1318.

Zeppernick, F., Meinhold-Heerlein, I., Shih, I. M. J. J. o. O., and Research, G. (2015). Precursors of ovarian cancer in the fallopian tube: Serous tubal intraepithelial carcinoma-an update. 41, 6-11. 
Zhai, W., Comai, L. J. M., and biology, c. (2000). Repression of RNA polymerase I transcription by the tumor suppressor p53. 20, 5930-5938.

Zhang, X., Hong, S., Zhang, M., Cai, Q., Zhang, M., and Xu, C. J. N. (2018). Proteomic alterations of fibroblasts induced by ovarian cancer cells reveal potential cancer targets. $65,104-112$.

Zhao, G., Chen, J., Deng, Y., Gao, F., Zhu, J., Feng, Z., Lv, X., Zhao, Z. J. B., and communications, b. r. (2011). Identification of NDRG1-regulated genes associated with invasive potential in cervical and ovarian cancer cells. 408, 154-159.

Zhu, Y., Lin, J., Chen, J., Huang, Q., Shao, L., and Lai, M. J. Y. c. H. (2005). The expression of Staufen Gene in colorectal cancer. $27,705-709$.

\section{Figures}

A

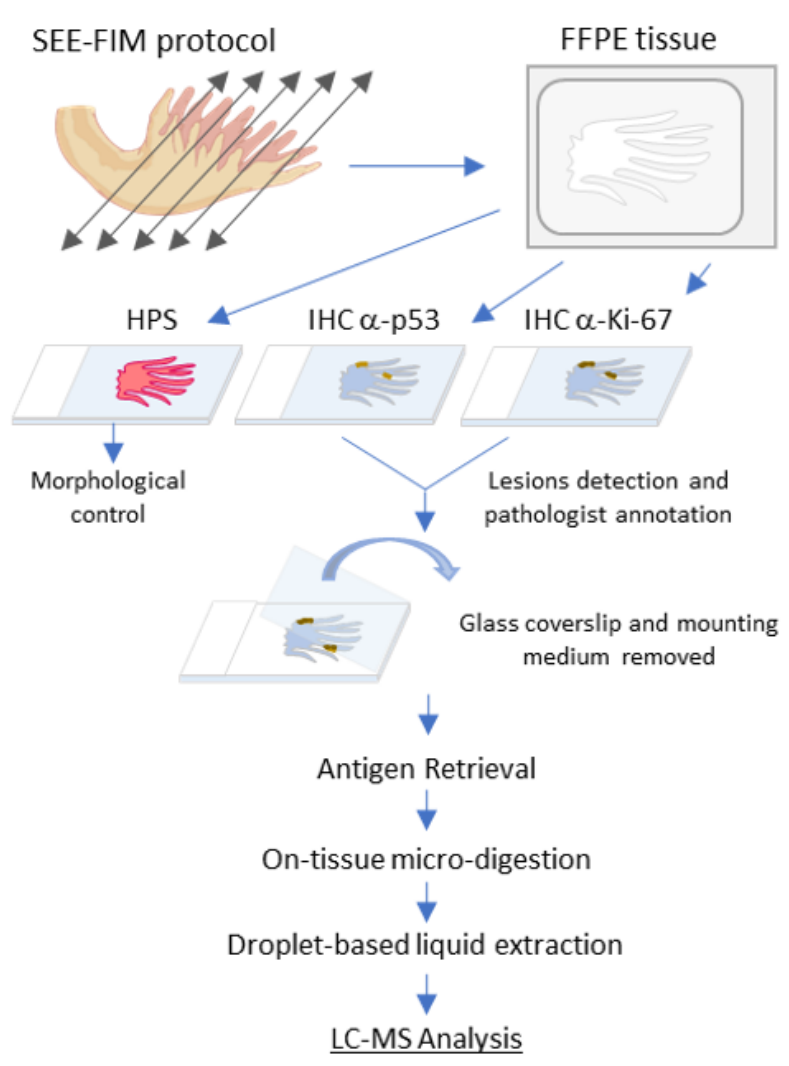

p53

B

p53 signature

STIL
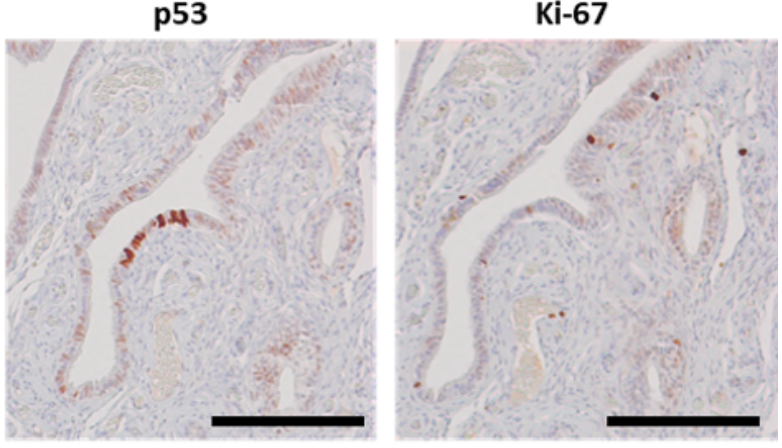

STIC
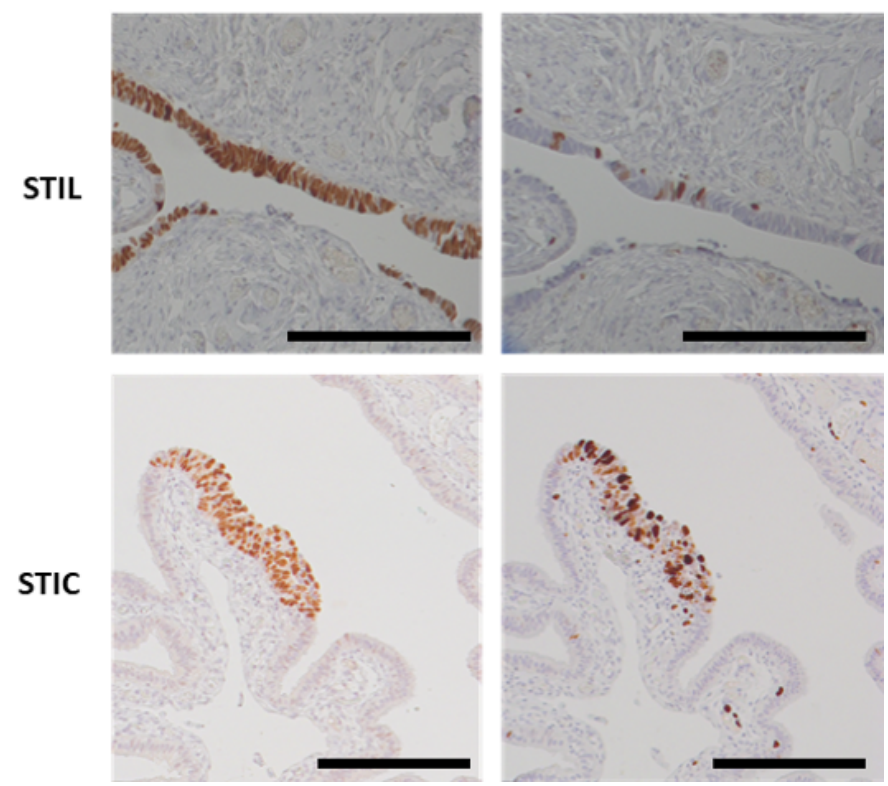

\section{Figure 1}

Workflow for spatially resolved proteomic using IHC tissue section. A) Protocol based on tissue from SEE-FIM protocol. After IHC against p53 and Ki-67, the coverslip glass and the mounting medium are removed to access the tissue section. A digestion of the lesion is performed directly on the tissue section and a droplet-based liquid extraction is performed to recover the peptides prior to MS-based proteomics analysis. B) Pre-neoplastic lesions found in the fallopian tube are defined by p53 positivity and Ki-67 index. For p53 signature: accumulation of p53 in 
at least 12 cells without morphological abnormalities and low Ki-67 index; STIL: same accumulation of p53 in more than 20 cells with some morphological abnormalities and an higher Ki-67 proliferating index (10-40\%); STIC: high p53 and Ki-67 index and cells atypical morphology (carcinoma-like).
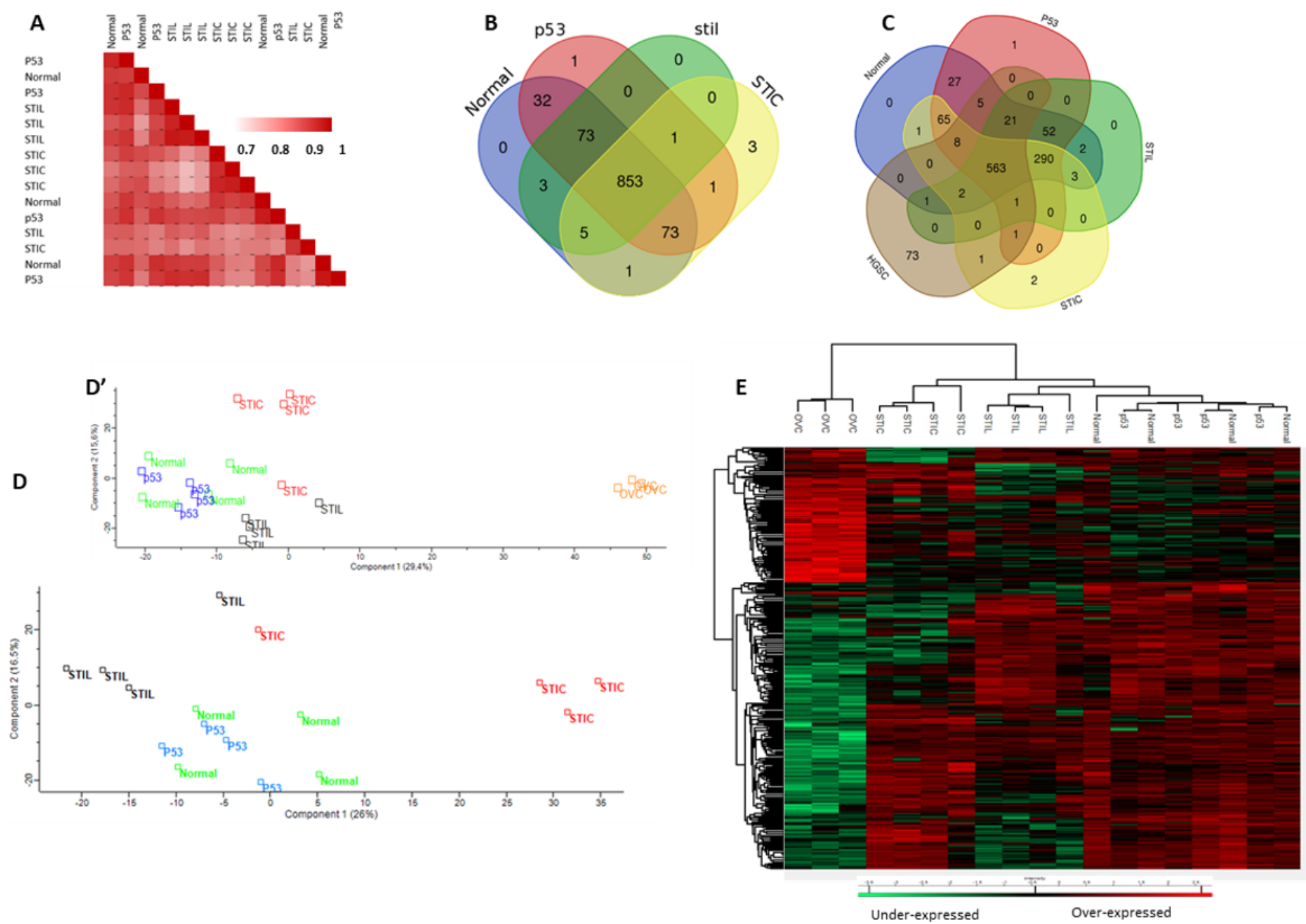

Figure 2

Comparison of the different lesions based on their proteomic signature. A) Correlation analysis based on the whole proteome. Ven diagrams showing distribution of identified proteins among B) normal tissue and the different lesions and C) with addition of HGSC data. Principal component analysis using D) normal tissue and the preneoplastic lesions and D') with addition to HGSC. E) Hierarchical clustering of the most variable proteins (ANOVA with permutation-based FDR < 0.05) including HGSC data 
A
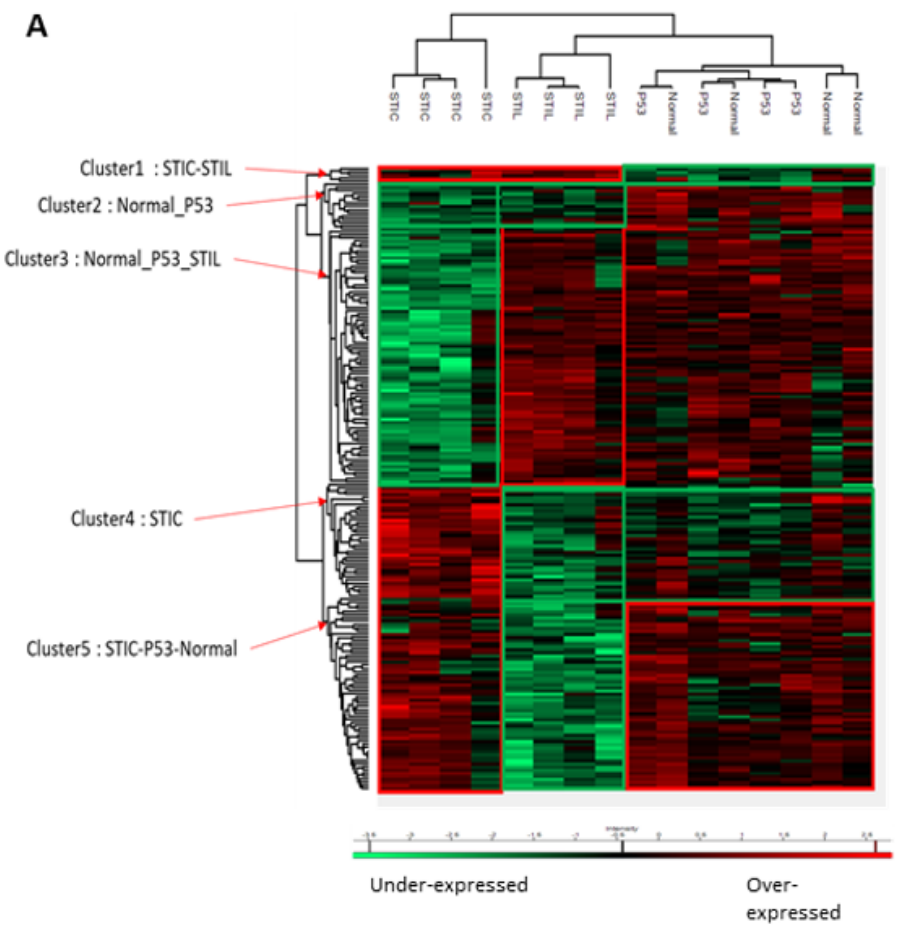

c) Cluster 4: STIC

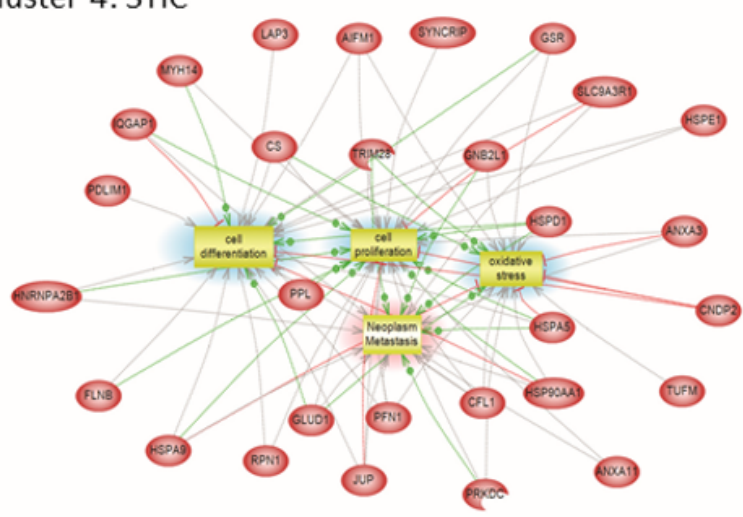

B

a) Cluster 2: normal-p53

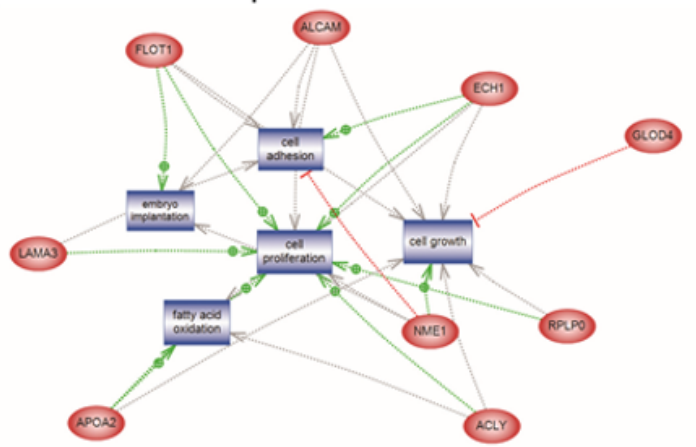

b) Cluster 3: normal-p53/STIL

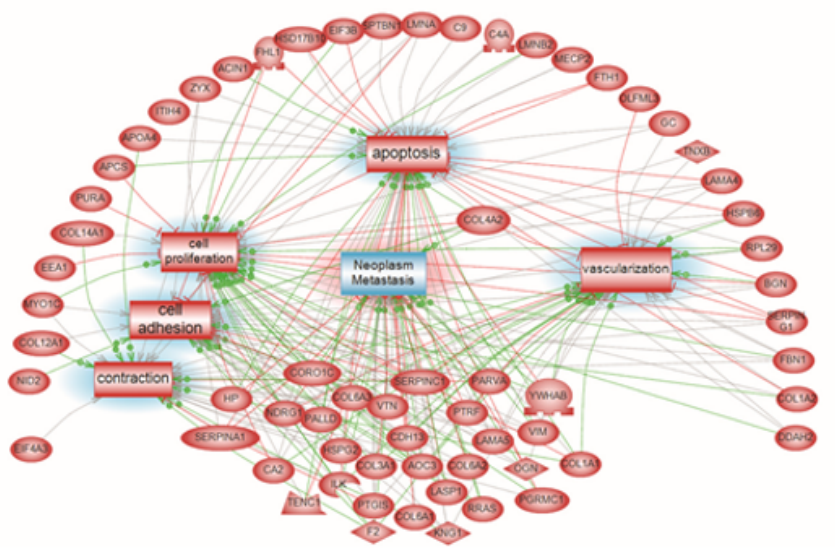

d) Cluster 5: normal-p53/STIC

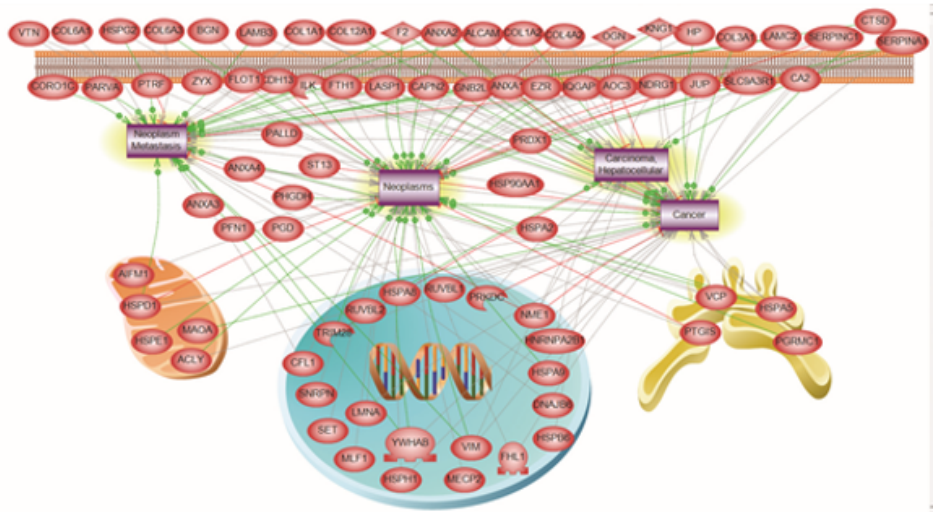

\section{Figure 3}

Proteomic analysis of the pre-neoplastic lesions. A) Hierarchical clustering of the most variable proteins between normal tissue, p53 signature, STIL and STIC ( $n=4$ for each category, ANOVA with permutation-based FDR $<0.05)$; B) Subnetwork Enrichment Analysis was done to highlight altered biological and functional pathways in the different clusters of proteins. 
A

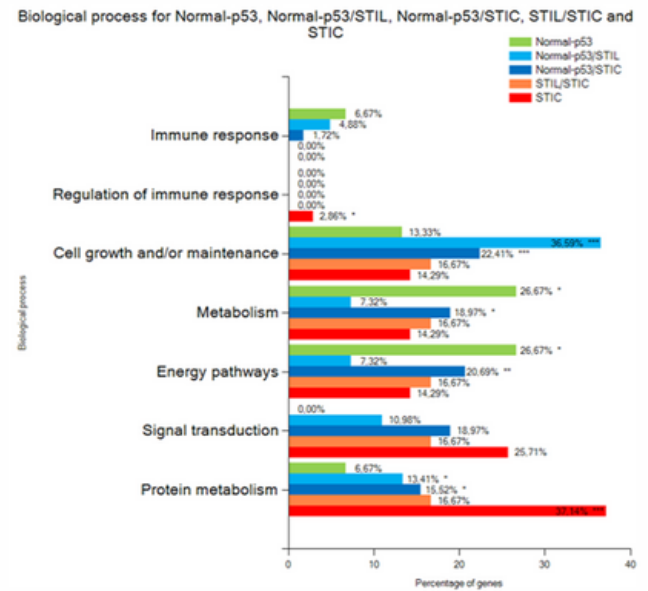

c

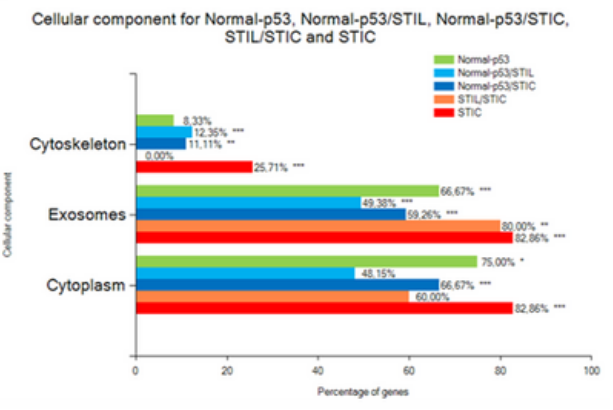

E

Biological pathway for normal-p53, STIL and STIC

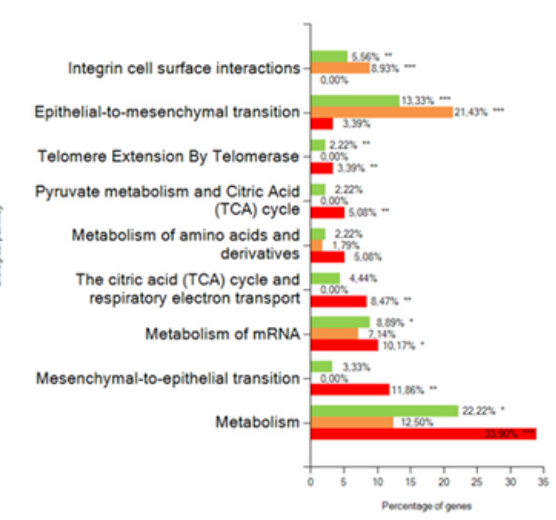

B

Transeription factor for Normal-p53, Normal-p53/STLL, Normal-p53/STIC, STILSTIC and

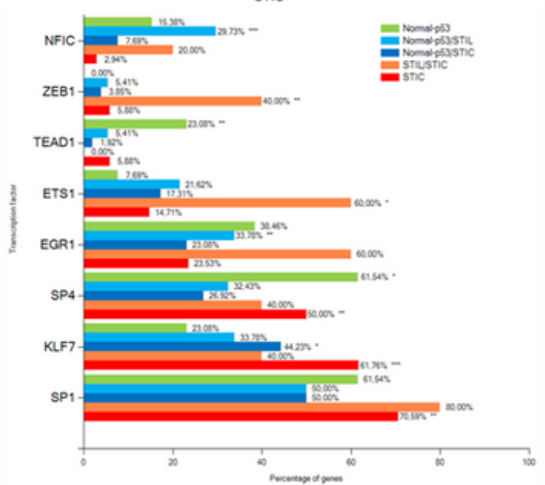

D

Biological process for normal-p53, STIL and STIC

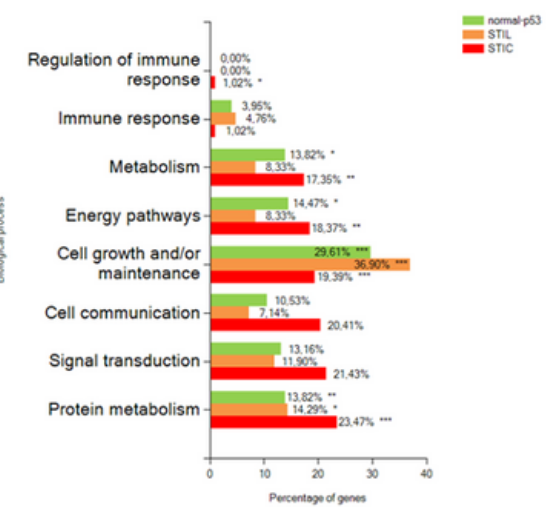

F

Molecular function for normal-p53, STIL and STIC

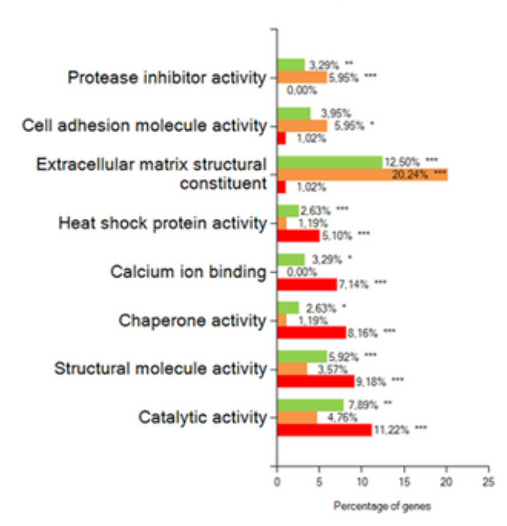

Figure 4

Annotation analysis of gene ontology terms. A) Biological process, B) Transcription factor and C) Cellular component for the clusters of proteins. D) Biological process, E) Biological pathway and F) Molecular function of the proteins overexpressed in each lesion. (hypergeometric test against all annotated gene/protein list of Funrich database, $p$-value is represented by stars: ${ }^{* \star} p<0.001$, ${ }^{* \star} p<0.01,{ }^{*} p<0.05$ no star for $p>0.05$ ). 
A

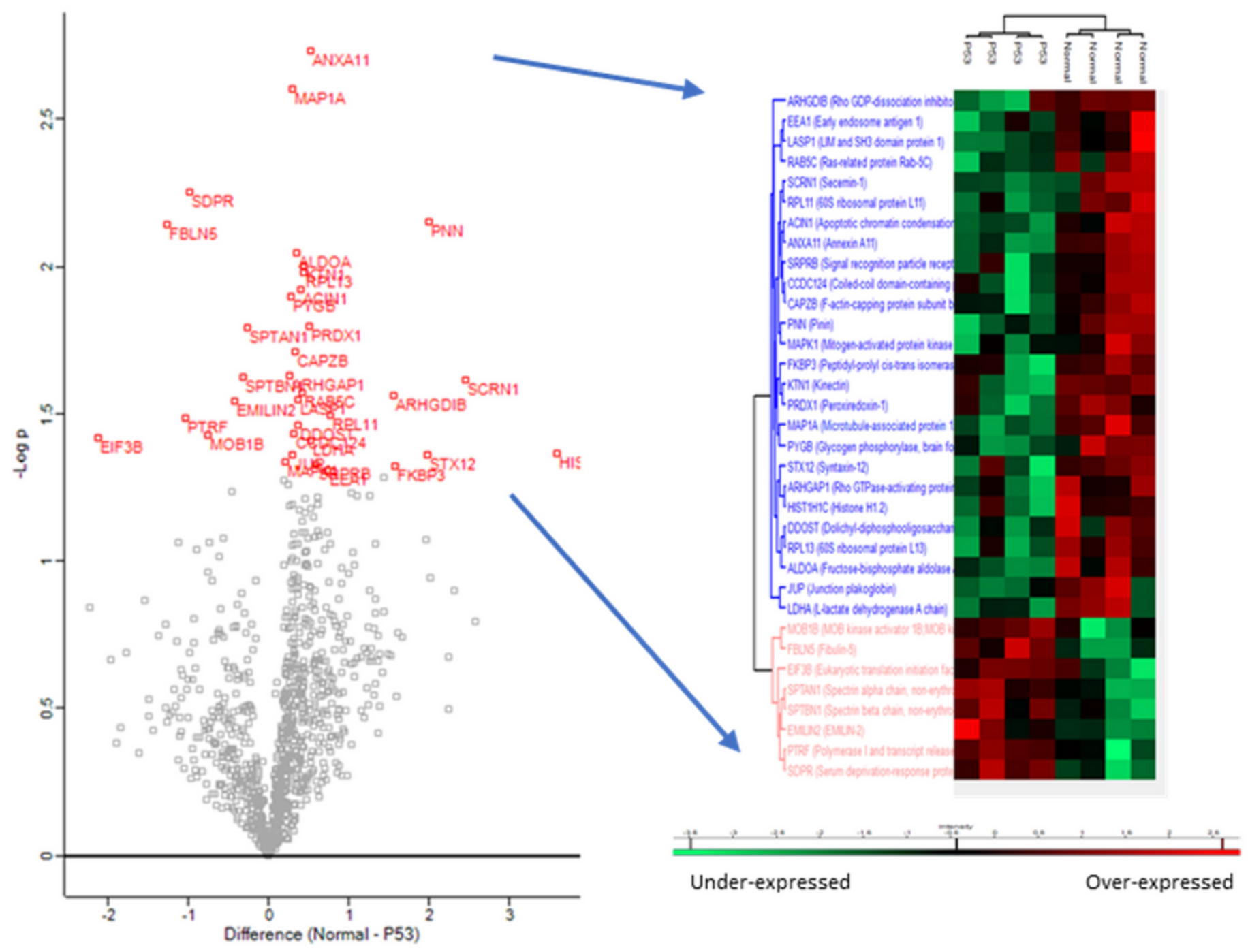

\section{Figure 5}

Comparison between p53 signature and normal tissue. A) Visualization of a t-test in form of a volcano plot comparing normal to $p 53$ lesion (proteins with $p<0.05$ in red.) B) Hierarchical clustering of the significant variable proteins between normal tissue and p53 signature (t-test with $p<0.05)$. 
A Polyubiquitin-C c.368G $>$ C
p.G123A|IFAAKQL|Missense|COSMIC|Breast

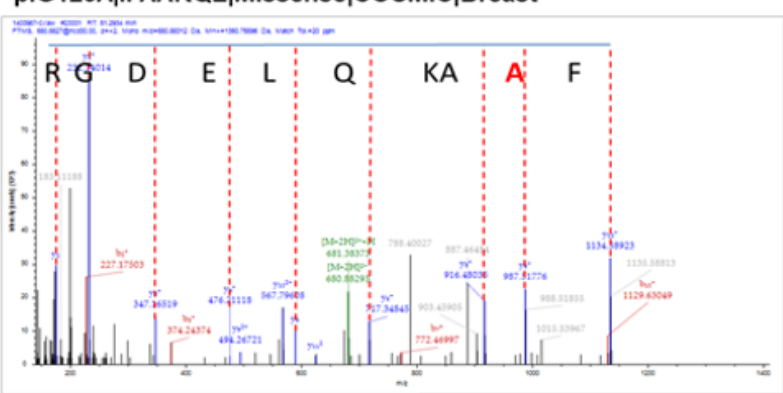

Histone H3.1 c.368A>G

p.K123R|IMPRDIQ|Missense|COSMIC|Endometrium(1)

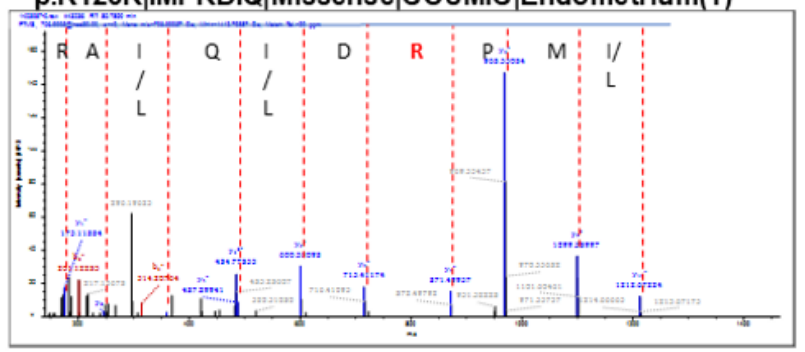

B

a

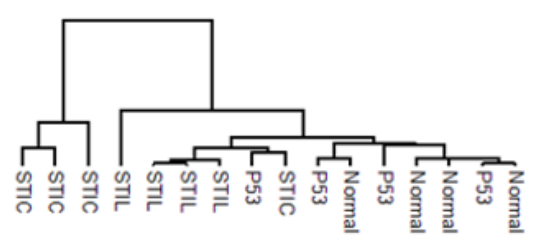

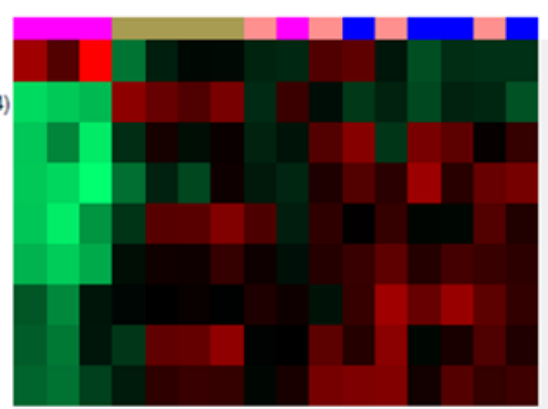

b

Protein GC (Vitamin D Binding Protein) C.1296T>G

p.D432E|KLPEATP|Missense|COSMIC|Stomach

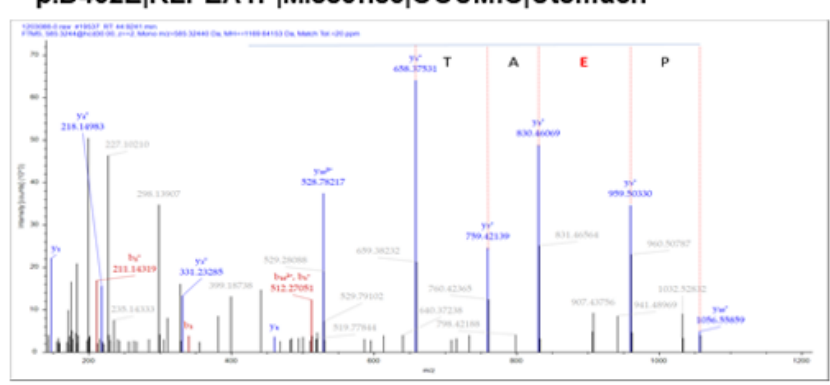

Histone H2B type 1-B c.169T>A

p.S57T|GISTKAM|Missense |COSMIC|Endometrium

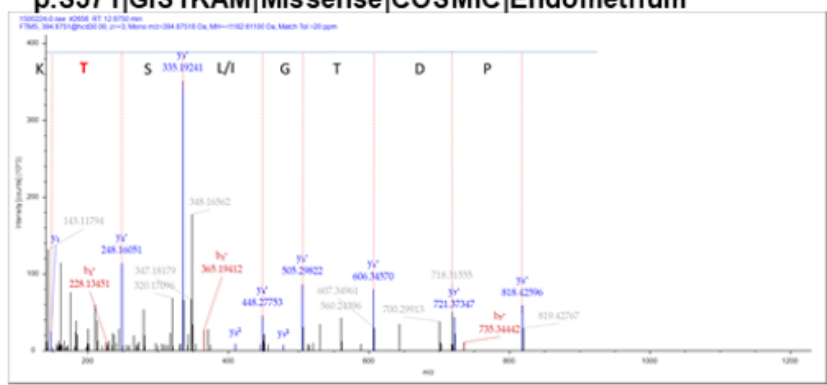

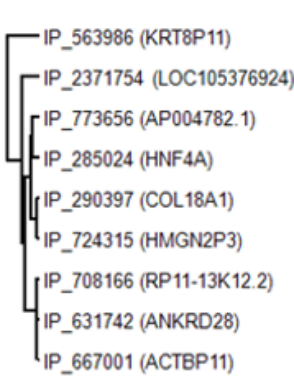

Figure 6

Potential mutations and Alternative proteins analysis. A) Annotated MS/MS spectra showing the mutated amino acid in red. B) Hierarchical clustering of the most variable alternative proteins between the lesion (a) or with adjunction of HGSC (b).

\section{Supplementary Files}

This is a list of supplementary files associated with this preprint. Click to download.

- Supp.Data1Specificproteinvenndiagram.xlsx

- Supp.Data2venndiagramHGSC.xIsx 
- Supp.Data3Heatmapcluster.xlsx

- Supp.Data4PantherDb.xlsx

- Supp.Data5p53vsNormal.xlsx

- Supp.Data6mutation.xlsx

- Supp.Data7Altprot.xlsx

- Supplementaryfigures.docx 